\title{
Fermion and meson mass generation in non-Hermitian Nambu-Jona-Lasinio models
}

\author{
Alexander Felski ${ }^{*}$ and S. P. Klevansky $\oplus^{\dagger}$ \\ Institut für Theoretische Physik, Universität Heidelberg, Philosophenweg 12, 69120 Heidelberg, Germany
}

(Received 28 January 2021; accepted 22 February 2021; published 12 March 2021)

\begin{abstract}
We investigate the effects of non-Hermiticity on interacting fermionic systems. We do this by including non-Hermitian bilinear terms into the $3+1$ dimensional Nambu-Jona-Lasinio (NJL) model. Two possible bilinear modifications give rise to $\mathcal{P} \mathcal{T}$-symmetric theories; this happens when the standard NJL model is extended either by a pseudovector background field $i g \bar{\psi} \gamma_{5} B_{\mu} \gamma^{\mu} \psi$ or by an antisymmetric-tensor background field $g \bar{\psi} F_{\mu \nu} \gamma^{\mu} \gamma^{\nu} \psi$. The three remaining bilinears are anti- $\mathcal{P} \mathcal{T}$-symmetric in nature, $i g \bar{\psi} B_{\mu} \gamma^{\mu} \psi, i g \bar{\psi} \gamma_{5} \psi$ and $i g \bar{\psi} \rrbracket \psi$, so that the Hamiltonian then has no overall symmetry. The pseudovector $i g \bar{\psi} \gamma_{5} B_{\mu} \gamma^{\mu} \psi$ and the vector $i g \bar{\psi} B_{\mu} \gamma^{\mu} \psi$ combinations, are, in addition, chirally symmetric. Thus, within this framework we are able to examine the effects that the various combinations of non-Hermiticity, $\mathcal{P} \mathcal{T}$ symmetry, chiral symmetry, and the two-body interactions of the NJL model have on the existence and dynamical generation of a real effective fermion mass (a feature which is absent in the corresponding modified massless free Dirac models) as well as on the masses of the composite particles, the pseudoscalar and scalar mesonic modes ( $\pi$ and $\sigma$ mesons). Our findings demonstrate that $\mathcal{P} \mathcal{T}$ symmetry is not necessary for real fermion mass solutions to exist; rather, the two-body interactions of the NJL model supersede the non-Hermitian bilinear effects. The effects of chiral symmetry are evident most clearly in the meson modes, the pseudoscalar of which will always be Goldstone in nature if the system is chirally symmetric. Second solutions of the mesonic equations are also discussed.
\end{abstract}

DOI: 10.1103/PhysRevD.103.056007

\section{INTRODUCTION}

The occurrence of real eigenvalue spectra in nonHermitian systems that are symmetric under combined parity reflection $\mathcal{P}$ and time reversal $\mathcal{T}$ has, since its first demonstration by Bender and Boettcher in 1998 [1], inspired a wide variety of theoretical and experimental studies in $\mathcal{P} \mathcal{T}$-symmetric physics. While initially focusing on classical and semiclassical systems, these studies were soon extended to include bosonic field theories as well [2]. For fermionic systems, Jones-Smith et al. [3] brought attention to the property of odd time-reversal symmetry $\left(\mathcal{T}^{2}=-\mathbb{1}\right)$ in $3+1$ dimensions and its importance in the context of $\mathcal{P} \mathcal{T}$ symmetry, in contrast to the even timereversal symmetry of bosonic systems.

In further studies involving fermions, unexpected behavior has emerged: In [4] the free Dirac Lagrangian was modified by the addition of non-Hermitian, but

\footnotetext{
felski@thphys.uni-heidelberg.de

spk@physik.uni-heidelberg.de
}

Published by the American Physical Society under the terms of the Creative Commons Attribution 4.0 International license. Further distribution of this work must maintain attribution to the author(s) and the published article's title, journal citation, and DOI. Funded by SCOAP ${ }^{3}$.
$\mathcal{P} \mathcal{T}$-symmetric, bilinears of the fermionic field $\psi$ and its conjugate $\bar{\psi}$. It was found that an unbroken $\mathcal{P} \mathcal{T}$ symmetry phase, that is, a regime with a real spectrum, does not simply exist: without a finite bare mass these modified Dirac fermions generally have complex physical masses. However, including higher-order interactions can override this breakdown of an unbroken $\mathcal{P} \mathcal{T}$ symmetry regime. We demonstrated in [5] that a region of real mass solutions exists, when the bilinear term that includes the $\mathcal{P} \mathcal{T}$ symmetric pseudovector background field $i g \bar{\psi} \gamma_{5} B_{\mu} \gamma^{\mu} \psi$ is incorporated into the $3+1$ dimensional Nambu-JonaLasinio (NJL) model, which contains two-body interactions as well. The gap equation of this modified system with Hamiltonian density

$$
\begin{aligned}
\mathcal{H}= & \bar{\psi}\left(-i \gamma^{k} \partial_{k}+m_{0}+i g \gamma_{5} B_{\mu} \gamma^{\mu}\right) \psi-G\left[(\bar{\psi} \psi)^{2}\right. \\
& \left.+\left(\bar{\psi} i \gamma_{5} \vec{\tau} \psi\right)^{2}\right]
\end{aligned}
$$

has real physical mass solutions even in the chiral limit of vanishing bare mass $m_{0}$, provided the coupling constant $g$ of the non-Hermitian bilinear does not exceed a critical value $g_{\text {crit }}$. Moreover, when compared to the standard NJL model $(g=0)$, increased fermion masses could be generated dynamically, mimicking the effect of a small bare mass $m_{0}$ without breaking chiral symmetry. 
Similar results have been demonstrated in [6-8] for models with Yukawa-type interactions that couple different fields, and can at least, in principle, be obtained from fourpoint interactions through partial bosonization.

The existence of an unbroken $\mathcal{P} \mathcal{T}$ symmetry regime in the modified NJL model (1) without a bare mass $m_{0}$ is remarkable, given that in the underlying modified Dirac theory $(G=0)$ this symmetry is realized in the broken regime exclusively. It raises the question as to whether the addition of a non-Hermitian bilinear has to uphold $\mathcal{P} \mathcal{T}$ symmetry at all. We thus ask what specific role the combination of chiral symmetry, $\mathcal{P} \mathcal{T}$ symmetry, and higher-order interactions play in the generation of real masses within fermionic models. The first aim of this study is to expand the analysis of the modified NJL model (1) to cover all possible non-Hermitian bilinear extensions, including but not limited to the only other $\mathcal{P} \mathcal{T}$-symmetric case of an antisymmetric-tensor background field $g \bar{\psi} F_{\mu \nu} \gamma^{\mu} \gamma^{\nu} \psi$ [4]. The analysis of these modifications to the NJL model furthermore covers cases in which chiral symmetry is preserved as well as cases in which it is broken explicitly.

Our second aim in this paper goes beyond the discussion of fermionic mass generation to study the effects on the mesonic composite states that can be generated within the context of these models. For those systems in which we find dynamically generated real fermion masses, we investigate the effect that the non-Hermitian bilinear terms have on the mass of the scalar and pseudoscalar bound states; i.e., these are the $\sigma$ and $\pi$ mesons in the context of quantum chromodynamics (QCD).

This paper is structured as follows: In Sec. II the modified NJL model is introduced and all possible bilinear additions leading to a non-Hermitian model are identified. Their behavior under $\mathcal{P} \mathcal{T}$ symmetry and chiral symmetry is presented. In Sec. III we first recapitulate the algebraic form of the gap equation and its solution for the system previously studied in [5]. We then proceed to analyze the structure of the gap equations and the existence of real fermion mass solutions for the other non-Hermitian extensions of the NJL model. In Sec. IV we investigate the corresponding mass of the scalar and pseudoscalar bound states for models in which a dynamical fermion mass generation could be identified. We conclude in Sec. V.

\section{THE MODIFIED NJL MODEL}

In its two-flavor version $\left(N_{f}=2\right)$, the standard NJL model [9] is given by the Hamiltonian density

$$
\mathcal{H}_{\mathrm{NJL}}=\bar{\psi}\left(-i \gamma^{k} \partial_{k}+m_{0}\right) \psi-G\left[(\bar{\psi} \psi)^{2}+\left(\bar{\psi} i \gamma_{5} \vec{\tau} \psi\right)^{2}\right],
$$

in terms of the Dirac matrices $\gamma$ with $k=1 \ldots 3$, the isospin SU(2) matrices $\vec{\tau}$, a coupling strength $G$, and the bare mass $m_{0}$. While the mass term $m_{0}$ breaks chiral symmetry explicitly, the specific combination of four-point interaction terms preserves it. In the limit of vanishing bare mass the model can thus be used to study the spontaneous breaking of chiral symmetry occurring through a mechanism that parallels pair condensation in the Bardeen-CooperSchrieffer theory of superconductivity [10]. As such it has been developed into an effective field theory of QCD in the chiral limit. In this context, including a small bare mass $m_{0}$ leads to a model with an approximate spontaneously broken chiral symmetry that has a small current quark mass and a light pseudo-Goldstone boson, identified as the pion. The numerical analysis throughout this paper will be based on established quantities within the QCD interpretation, using a four-momentum Euclidean cutoff scale of $\Lambda=$ $1015 \mathrm{MeV}$ for the purpose of regularization and a coupling strength satisfying $G \Lambda^{2}=3.93$, cf. [11].

In continuation of our previous study [5], we introduce the bilinear extension of the NJL model

$$
\mathcal{H}=\bar{\psi}\left(-i \gamma^{k} \partial_{k}+m_{0}+g \Gamma\right) \psi-G\left[(\bar{\psi} \psi)^{2}+\left(\bar{\psi} i \gamma_{5} \vec{\tau} \psi\right)^{2}\right],
$$

where $\Gamma$ generally denotes a complex $4 \times 4$ matrix and $g$ is the associated real coupling constant. In the following, we consider the complete set of $4 \times 4$ matrices generated by the Dirac matrices and identify all those that are nonHermitian, irrespective of whether they are $\mathcal{P} \mathcal{T}$ symmetric or not. The matrix $\Gamma$ in (2) will then take on the corresponding role in the subsequent analysis. In addition, we consider the behavior of the non-Hermitian bilinears under chiral symmetry transformations, and thus infer the symmetries present in the modified NJL models given by (2).

Any bilinear of the fermionic field $\psi$ and its conjugate $\bar{\psi}$ is of the form $\bar{\psi} \Gamma \psi$. As such, they can be written as real superpositions based on the following 32 matrices:

$$
\begin{aligned}
& \mathbb{1}, \quad \gamma_{5}, \quad B_{\mu} \gamma^{\mu}, \quad \gamma_{5} B_{\mu} \gamma^{\mu}, \quad F_{\mu \nu} \gamma^{\mu} \gamma^{\nu}, \\
& i \mathbb{1}, \quad i \gamma_{5}, \quad i B_{\mu} \gamma^{\mu}, \quad i \gamma_{5} B_{\mu} \gamma^{\mu}, \quad i F_{\mu \nu} \gamma^{\mu} \gamma^{\nu} \text {, }
\end{aligned}
$$

where $\mu \leq \nu$ denote spin indices, $B_{\mu}$ are real vector elements, and $F_{\mu \nu}$ are real elements of an antisymmetric matrix. The gamma matrix structure in the bilinears $\bar{\psi} \Gamma \psi$ formed by these behave as scalars, pseudoscalars, vectors, pseudovectors, and antisymmetric second-rank tensors, respectively, under observer Lorentz transformations [12-14] (from left to right). Including an imaginary unit in any bilinear will change it from being symmetric to being antisymmetric under Hermitian conjugation, and vice versa. Thus, we identify half of the bilinear terms associated with the matrices in (3) to be anti-Hermitian, namely, those for which $\Gamma$ takes one of the forms

$$
i \mathbb{1}, \quad \gamma_{5}, \quad i B_{\mu} \gamma^{\mu}, \quad i \gamma_{5} B_{\mu} \gamma^{\mu}, \quad F_{\mu \nu} \gamma^{\mu} \gamma^{\nu}
$$


These terms result in the non-Hermitian extensions of the NJL model considered throughout this paper.

To determine the behavior of $\bar{\psi} \Gamma \psi$, with $\Gamma$ being one of the terms in (4), under combined parity reflection $\mathcal{P}$ and time reversal $\mathcal{T}$, recall the usual definition of these transformations in $3+1$ dimensions [15]:

$$
\begin{gathered}
\mathcal{P}: \psi(t, \mathbf{x}) \rightarrow \mathcal{P} \psi(t, \mathbf{x}) \mathcal{P}^{-1}=\gamma^{0} \psi(t,-\mathbf{x}), \\
\mathcal{T}: \psi(t, \mathbf{x}) \rightarrow \mathcal{T} \psi(t, \mathbf{x}) \mathcal{T}^{-1}=i \gamma^{1} \gamma^{3} \psi^{*}(-t, \mathbf{x}) .
\end{gathered}
$$

We find that only two types of non-Hermitian bilinears are $\mathcal{P} \mathcal{T}$ symmetric; specifically, $[\mathcal{P} \mathcal{T}, \Gamma]=0$ for

$$
\begin{aligned}
& \Gamma_{P T_{1}}=i \gamma_{5} B_{\mu} \gamma^{\mu}, \\
& \Gamma_{P T_{2}}=F_{\mu \nu} \gamma^{\mu} \gamma^{\nu} .
\end{aligned}
$$

The remaining non-Hermitian terms are anti- $\mathcal{P} \mathcal{T}$ symmetric, $\{\mathcal{P} \mathcal{T}, \Gamma\}=0$, and will in the following be referred to as

$$
\begin{gathered}
\Gamma_{a P T_{1}}=i B_{\mu} \gamma^{\mu}, \\
\Gamma_{a P T_{2}}=\gamma_{5}, \\
\Gamma_{a P T_{3}}=i \mathbb{1} .
\end{gathered}
$$

Notice that in these anti- $\mathcal{P} \mathcal{T}$-symmetric cases the Hamiltonian density (2) has no such overall symmetry. The system as a whole is thus non-Hermitian and non- $\mathcal{P} \mathcal{T}$ symmetric.

Moreover, only two of the terms in (4) anticommute with $\gamma_{5}$, leading to an axial flavor symmetry and thus an overall chiral symmetry of the Hamiltonian density (2) in the limit of vanishing bare mass: $\Gamma_{P T_{1}}$ and $\Gamma_{a P T_{1}}$. In contrast, even without a bare mass $m_{0}$, chiral symmetry is broken explicitly in the theories based on including the terms $\Gamma_{P T_{2}}, \Gamma_{a P T_{2}}$, and $\Gamma_{a P T_{3}}$.

Thus, we have identified five possible non-Hermitian bilinear extensions of the NJL model: One that is $\mathcal{P} \mathcal{T}$ symmetric and chirally symmetric $\left(\Gamma_{P T_{1}}\right)$, one that is $\mathcal{P} \mathcal{T}$ symmetric but breaks chiral symmetry explicitly $\left(\Gamma_{P T_{2}}\right)$, one that is anti- $\mathcal{P} \mathcal{T}$ symmetric but chirally symmetric $\left(\Gamma_{a P T_{1}}\right)$, and two that break both $\mathcal{P} \mathcal{T}$ and chiral symmetry $\left(\Gamma_{a P T_{2}}\right.$ and $\left.\Gamma_{a P T_{3}}\right)$.

\section{GAP EQUATION AND FERMION MASSES}

A self-consistent approximation to the effective fermion mass of the standard NJL model is expressed by the gap equation, which can be obtained following FeynmanDyson perturbation theory, cf. $[11,16]$. We established in [5] that the gap equation of an extended NJL model such as (2), which includes additional bilinear terms, has the same general structure as that of the standard NJL model:

$$
m=m_{0}+\frac{2 i G N_{c} N_{f}}{(2 \pi)^{4}} I,
$$

where $m$ denotes the effective fermion mass, $N_{c}=3$ the number of colors, $N_{f}=2$ is the number of flavors, and $I$ is the momentum integral over the spinor trace of the fermion propagator:

$$
I=\int d^{4} p \operatorname{tr}[S(p)]
$$

Here, the full fermion propagator $S(p)$ has the same form as the propagator $S^{0}(p)$ of the corresponding modified Dirac theory (obtained at $G=0$ ) and can be found by replacing the bare mass $m_{0}$ with the effective mass $m$ :

$$
S(p)=(\not p-m-g \Gamma)^{-1} .
$$

Analyzing the effective fermion mass of the non-Hermitian NJL models introduced in the previous section thus relies on the evaluation of the spinor trace and the momentum integration in (13) for these models.

In this section we derive the algebraic gap equation for the five non-Hermitian NJL models and study the existence and behavior of real fermion mass solutions. Section III A summarizes the results for the $\mathcal{P} \mathcal{T}$-symmetric model based on $\Gamma_{P T_{1}}$, which were previously obtained in [5]. In Sec. III B the second $\mathcal{P} \mathcal{T}$-symmetric case (based on $\Gamma_{P T_{2}}$ ) is analyzed, and Secs. III C-IIIE discuss the three remaining non- $\mathcal{P} \mathcal{T}$-symmetric models.

$$
\text { A. } \Gamma_{P T_{1}}=i \gamma_{5} B_{\mu} \gamma^{\mu}
$$

In the case of the $\mathcal{P} \mathcal{T}$-symmetric model based on including the pseudovector background field $\Gamma_{P T_{1}}=$ $i \gamma_{5} B_{\mu} \gamma^{\mu}$, as studied in [5], the full fermion propagator (14) was rationalized,

$$
S(p)=\frac{\left(\not p+m+i \gamma_{5} B_{\mu} \gamma^{\mu}\right)\left[\left(p^{2}-m^{2}-g^{2} B^{2}\right)+2 i g m \gamma_{5} B_{\nu} \gamma^{\nu}+2 i g B_{\nu} p^{\nu} \gamma_{5}\right]}{\left(p^{2}-m^{2}-g^{2} B^{2}\right)^{2}-4 g^{2} m^{2} B^{2}+4 g^{2}\left(B_{\mu} p^{\mu}\right)^{2}},
$$

in order to evaluate the spinor trace

$$
\operatorname{tr}[S(p)]=\frac{4 m\left(p^{2}-m^{2}+g^{2} B^{2}\right)}{\left(p^{2}-m^{2}-g^{2} B^{2}\right)^{2}-4 g^{2} m^{2} B^{2}+4 g^{2}\left(B_{\mu} p^{\mu}\right)^{2}} .
$$


The introduction of a Euclidean four-momentum cutoff $\Lambda$ for the purpose of regularization then allows the momentum integration in (13). To that end we change to Euclidean coordinates with $p_{0}=i p_{4}$ and $B_{0}=i B_{4}$, such that $p^{2}=-p_{E}^{2}$, $B^{2}=-B_{E}^{2}$, and $B_{\mu} p^{\mu}=-B_{E} \cdot p_{E}$. In the chiral limit of vanishing bare mass $m_{0}$, the gap equation (12) then takes on the algebraic form

$$
\begin{aligned}
\frac{2 \pi^{2}}{\tilde{G} N_{c} N_{f}}= & \frac{1}{4 \tilde{g}^{2}}\left\{\sqrt{\left(1+\tilde{m}^{2}-\tilde{g}^{2}\right)^{2}+4 \tilde{g}^{2} \tilde{m}^{2}}\left(1+\tilde{m}^{2}+7 \tilde{g}^{2}\right)-\left(\tilde{m}^{2}+\tilde{g}^{2}\right)\left(2+\tilde{m}^{2}+7 \tilde{g}^{2}\right)-1\right. \\
& \left.+4 \tilde{g}^{2}\left(2 \tilde{g}^{2}-\tilde{m}^{2}\right) \ln \left[\frac{1}{2 \tilde{m}^{2}}\left(\sqrt{\left(1+\tilde{m}^{2}-\tilde{g}^{2}\right)^{2}+4 \tilde{g}^{2} \tilde{m}^{2}}+1+\tilde{m}^{2}-\tilde{g}^{2}\right)\right]\right\}
\end{aligned}
$$

in terms of the rescaled parameters $\tilde{G}=G \Lambda^{2}, \tilde{m}=m \Lambda^{-1}$, and $\tilde{g}=g\left|B_{E}\right| \Lambda^{-1}$, which is proportional to the amplitude of the pseudovector background field.

In the context of $\mathrm{QCD}$, this equation has real fermion mass solutions $m$ as long as $\tilde{g}<\tilde{g}_{\text {crit }} \approx 1.261$. The behavior of the solution in this regime is shown in Fig. 1. The largest mass of $m \approx 460.870 \mathrm{MeV}$ is found at a coupling value of $\tilde{g} \approx 0.702$. Notice that masses larger than the $m_{\mathrm{NJL}} \approx$ $238.487 \mathrm{MeV}$ of the standard NJL model are dynamically generated for all coupling values below $\tilde{g}_{d y n} \approx 1.183$. A current quark mass in the range of the bare up quark mass, $m_{u}=(1.7-3.3) \mathrm{MeV}$, is already generated at small coupling values $\tilde{g} \approx(0.025-0.034)$ and in the range of the bare down quark mass, $m_{d}=(4.1-5.8) \mathrm{MeV}$ for $\tilde{g} \approx(0.038-0.046)$.

Altogether, the $\mathcal{P} \mathcal{T}$ and chirally symmetric modified NJL model based on $\Gamma_{P T_{1}}$ admits a finite region of unbroken $\mathcal{P} \mathcal{T}$ symmetry, even for vanishing bare mass $m_{0}$. Real fermion mass solutions are obtained for coupling constant values up to a critical value $\tilde{g}_{\text {crit }}$. Fermion mass is dynamically generated for $\tilde{g}<\tilde{g}_{d y n}<\tilde{g}_{\text {crit }}$; larger coupling values lead to an effective mass loss.

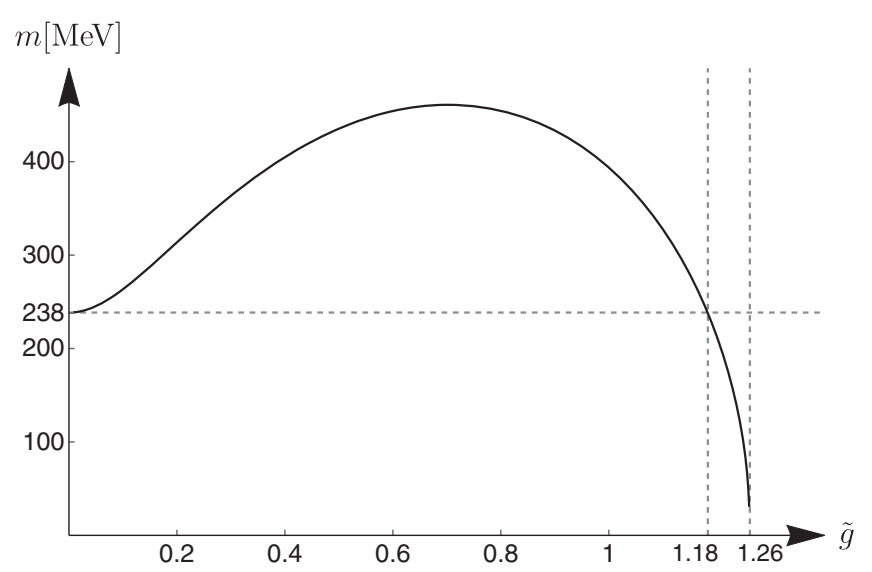

FIG. 1. Behavior of the effective fermion mass $m$ in the modified NJL model based on $\Gamma_{P T_{1}}$ as a function of the scaled coupling constant $\tilde{g}=g\left|B_{E}\right| \Lambda^{-1}$.

\section{B. $\Gamma_{P T_{2}}=F_{\mu \nu} \gamma^{\mu} \gamma^{\nu}$}

For the $\mathcal{P} \mathcal{T}$-symmetric model based on $\Gamma_{P T_{2}}=F_{\mu \nu} \gamma^{\mu} \gamma^{\nu}$, where $F_{\mu \nu}=-F_{\nu \mu}$ is real, the first goal is to rationalize the full fermion propagator

$$
S(p)=\left(\not p-m-g F_{\mu \nu} \gamma^{\mu} \gamma^{\nu}\right)^{-1}
$$

in order to evaluate the spinor trace in (13). We expand $S(p)$ with $\left(\not p-m+g F_{\alpha \beta} \gamma^{\alpha} \gamma^{\beta}\right)$. The denominator then takes the form

$$
\begin{aligned}
& \left(\not p-m-g F_{\mu \nu} \gamma^{\mu} \gamma^{\nu}\right)\left(\not p-m+g F_{\alpha \beta} \gamma^{\alpha} \gamma^{\beta}\right) \\
& \quad=\left(p^{2}+m^{2}-2 g^{2} f_{1}\right)-2 m \not b-4 g F_{\mu \nu} p^{\nu} \gamma^{\mu}+8 i g^{2} f_{2} \gamma_{5},
\end{aligned}
$$

where we have used the relations $F_{\mu \nu}\left(\gamma^{\mu} \gamma^{\nu} \not p-\not p \gamma^{\mu} \gamma^{\nu}\right)=$ $4 F_{\mu \nu} p^{\nu} \gamma^{\mu}$ and $F_{\mu \nu} F_{\alpha \beta} \gamma^{\mu} \gamma^{\nu} \gamma^{\alpha} \gamma^{\beta}=2 f_{1} \mathbb{1}-8 i f_{2} \gamma_{5}$, with

$$
f_{1}=2\left(F_{01}^{2}+F_{02}^{2}+F_{03}^{2}-F_{12}^{2}-F_{13}^{2}-F_{23}^{2}\right)=\operatorname{tr}\left[F^{2}\right],
$$

$$
f_{2}=\left(F_{01} F_{23}-F_{02} F_{13}+F_{03} F_{12}\right)=\operatorname{Pf}(F) .
$$

The denominator still contains nonscalar contributions; thus, we expand $S(p)$ again with a factor that has the opposite sign in those terms: the denominator of the resulting expression becomes

$$
\begin{aligned}
& {\left[\left(p^{2}+m^{2}-2 g^{2} f_{1}\right)-2 m \not p-4 g F_{\mu \nu} p^{\nu} \gamma^{\mu}+8 i g^{2} f_{2} \gamma_{5}\right]} \\
& \quad \times\left[\left(p^{2}+m^{2}-2 g^{2} f_{1}\right)+2 m \not p+4 g F_{\alpha \beta} p^{\beta} \gamma^{\alpha}-8 i g^{2} f_{2} \gamma_{5}\right] \\
& =\left(p^{2}+m^{2}-2 g^{2} f_{1}\right)^{2}-4 m^{2} p^{2}+64 g^{4} f_{2}^{2} \\
& \quad+16 g^{2} p^{\mu} F_{\mu \nu} F_{\alpha}^{\nu} p^{\alpha},
\end{aligned}
$$

where we utilized $\left\{\gamma^{\mu}, \gamma^{\nu}\right\}=2 \eta^{\mu \nu}, \quad\left\{\gamma^{\mu}, \gamma_{5}\right\}=0$, and $F_{\mu \nu}=-F_{\nu \mu}$. It is now a scalar and the full propagator can then be written in the rationalized form 


$$
S(p)=\frac{\left(\not p-m+g F_{\mu \nu} \gamma^{\mu} \gamma^{\nu}\right)\left[\left(p^{2}+m^{2}-2 g^{2} f_{1}\right)+2 m \not p+4 g F_{\alpha \beta} p^{\beta} \gamma^{\alpha}-8 i g^{2} f_{2} \gamma_{5}\right]}{\left(p^{2}+m^{2}-2 g^{2} f_{1}\right)^{2}-4 m^{2} p^{2}+64 g^{4} f_{2}^{2}+16 g^{2} p^{\mu} F_{\mu \nu} F_{\alpha}^{\nu} p^{\alpha}} .
$$

In multiplying out the numerator, we notice that almost all resulting terms have a vanishing spinor trace. Using that $\operatorname{tr}[\mathbb{1}]=4, \operatorname{tr}\left[\gamma^{\mu} \gamma^{\nu}\right]=4 \eta^{\mu \nu}$, and $F_{\mu}{ }^{\mu}=0$ we find the trace of the full propagator to be

$$
\operatorname{tr}[S(p)]=\frac{4 m\left(p^{2}-m^{2}+2 g^{2} f_{1}\right)}{\left(p^{2}+m^{2}-2 g^{2} f_{1}\right)^{2}-4 m^{2} p^{2}+64 g^{4} f_{2}^{2}+16 g^{2} p^{\mu} F_{\mu \nu} F_{\alpha}^{\nu} p^{\alpha}} .
$$

For the momentum integration in (13) we now regularize the integral in the Euclidean four-momentum cutoff method. That is, we first change to a Euclidean system by denoting $p_{0}=i p_{4}$ and $F_{0 k}=i F_{4 k}, \forall k \in[1,3]$, such that $d^{4} p=i d^{4} p_{E}, p^{2}=-p_{E}^{2}$, and $p^{\mu} F_{\mu \nu} F_{\alpha}^{\nu} p^{\alpha}=$ $-p_{E} \cdot F_{E} \cdot F_{E} \cdot p_{E}$, where the (complex) matrix $F_{E}$ is

$$
F_{E}=\left[\begin{array}{cccc}
0 & F_{41} & F_{42} & F_{43} \\
-F_{41} & 0 & F_{12} & F_{13} \\
-F_{42} & -F_{12} & 0 & F_{23} \\
-F_{43} & -F_{13} & -F_{23} & 0
\end{array}\right] .
$$

In this form, the dependence on $F_{E}$ is somewhat unwieldy. When $F_{E}$ is diagonalizable, it is orthogonally so [17], such that a transformation $Q$ exists, with $Q^{T} Q=\mathbb{1}$ and $Q^{T} F_{E} Q=\operatorname{diag}\left(\lambda_{1},-\lambda_{1}, \lambda_{2},-\lambda_{2}\right)$, where

$$
\lambda_{1,2}=\frac{1}{2} \sqrt{f_{1} \mp \sqrt{f_{1}^{2}+16 f_{2}^{2}}} .
$$

Thus transforming the Euclidean four-momentum according to $p_{E} \rightarrow Q \cdot p_{E}$ leaves the integral measure invariant and results in a much more convenient dependence of (20) on the momentum components. This of course relies on the diagonalizability of $F_{E}$, which from the eigenvalues (22) we see to be so, provided that $f_{2}$ does not vanish. Since $f_{2}$ as given in (18) implies that $f_{2}^{2}=\operatorname{Det}(F)$, this is a minor restriction and will be considered to be the case in what follows. With the introduction of the four-momentum cutoff $\Lambda$, the regularized integral (13) over the trace (20) then has the form

$$
I_{P T_{2}}=-4 i m \int^{\Lambda} d^{4} p_{E} \frac{\left(p_{E}^{2}+m^{2}-2 g^{2} f_{1}\right)}{\left(p_{E}^{2}-m^{2}+2 g^{2} f_{1}\right)^{2}+4 m^{2} p_{E}^{2}+64 g^{4} f_{2}^{2}-16 g^{2} \lambda_{1}^{2}\left(p_{4}^{2}+p_{1}^{2}\right)-16 g^{2} \lambda_{2}^{2}\left(p_{2}^{2}+p_{3}^{2}\right)} .
$$

To evaluate this, we first express the momentum components in two sets of polar coordinates, where $p_{4}=R_{1} \cos \phi_{1}$, $p_{1}=R_{1} \sin \phi_{1}$ and $p_{2}=R_{2} \cos \phi_{2}, p_{3}=R_{2} \sin \phi_{2}$. Equation (23) becomes

$$
I_{P T_{2}}=-16 i \pi^{2} m \int^{\Lambda} d R_{1} d R_{2} \frac{R_{1} R_{2}\left(R_{1}^{2}+R_{2}^{2}+m^{2}-2 g^{2} f_{1}\right)}{\left(R_{1}^{2}+R_{2}^{2}-m^{2}+2 g^{2} f_{1}\right)^{2}+4 m^{2}\left(R_{1}^{2}+R_{2}^{2}\right)+64 g^{4} f_{2}^{2}-16 g^{2}\left(\lambda_{1}^{2} R_{1}^{2}+\lambda_{2}^{2} R_{2}^{2}\right)} .
$$

Now, combining $R_{1}$ and $R_{2}$ in polar coordinates $R_{1}=$ $r \cos \theta$ and $R_{2}=r \sin \theta$ with $\theta \in[0, \pi / 2]$ and $r \in[0, \Lambda]$ yields

$$
\begin{aligned}
I_{P T_{2}}= & -16 i \pi^{2} m \int_{0}^{\Lambda} d r r^{3}\left(r^{2}+m^{2}-2 g^{2} f_{1}\right) \\
& \times \int_{0}^{\pi / 2} d \theta \frac{\cos \theta \sin \theta}{A(r)-B(r) \cos ^{2} \theta}
\end{aligned}
$$

where

$$
\begin{aligned}
A(r)= & \left(r^{2}+m^{2}-2 g^{2} f_{1}^{2}\right)+64 g^{4} f_{2}^{2} \\
& +4 g^{2} r^{2}\left(f_{1}-\sqrt{f_{1}^{2}+16 f_{2}^{2}}\right),
\end{aligned}
$$

$$
B(r)=-8 g^{2} r^{2} \sqrt{f_{1}^{2}+16 f_{2}^{2}}
$$

The angular part is a standard integral [18], and $I_{P T_{2}}$ becomes

$$
I_{P T_{2}}=-i \pi^{2} m \int_{0}^{\Lambda} d r \frac{r\left(r^{2}+m^{2}-2 g^{2} f_{1}\right)}{g^{2} \sqrt{f_{1}^{2}+16 f_{2}^{2}}} \ln \left[1-\frac{B(r)}{A(r)}\right]
$$

and the remaining radial integration can then be rewritten as 


$$
I_{P T_{2}}=\frac{i \pi^{2} m}{2 g^{2} \sqrt{f_{1}^{2}+16 f_{2}^{2}}} \int_{0}^{\Lambda^{2}} d z\left(z+m^{2}-2 g^{2} f_{1}\right)\left\{\ln \left(z+a_{1}\right)+\ln \left(z+a_{2}\right)-\ln \left(z+a_{3}\right)-\ln \left(z+a_{4}\right)\right\},
$$

with

$$
\begin{aligned}
& a_{1,2}=m^{2}-2 g^{2} \sqrt{f_{1}^{2}+16 f_{2}^{2}} \pm \sqrt{4 m^{2} g^{2}\left(f_{1}-\sqrt{f_{1}^{2}+16 f_{2}^{2}}\right)}, \\
& a_{3,4}=m^{2}+2 g^{2} \sqrt{f_{1}^{2}+16 f_{2}^{2}} \pm \sqrt{4 m^{2} g^{2}\left(f_{1}+\sqrt{f_{1}^{2}+16 f_{2}^{2}}\right)} .
\end{aligned}
$$

After integration and some further simplification this then becomes

$$
\begin{aligned}
I_{P T_{2}}= & \frac{i \Lambda^{3} \pi^{2} \tilde{m}}{2 \tilde{g}^{2} \sqrt{1+f^{2}}}\left[\left(\tilde{a}_{1}+1\right) \ln \left(\frac{1+\tilde{a}_{1}}{\tilde{a}_{1}}\right)\left(\tilde{m}^{2}-2 \tilde{g}^{2}+\frac{1-\tilde{a}_{1}}{2}\right)+\left(\tilde{a}_{2}+1\right) \ln \left(\frac{1+\tilde{a}_{2}}{\tilde{a}_{2}}\right)\left(\tilde{m}^{2}-2 \tilde{g}^{2}+\frac{1-\tilde{a}_{2}}{2}\right)\right. \\
& \left.-\left(\tilde{a}_{3}+1\right) \ln \left(\frac{1+\tilde{a}_{3}}{\tilde{a}_{3}}\right)\left(\tilde{m}^{2}-2 \tilde{g}^{2}+\frac{1-\tilde{a}_{3}}{2}\right)-\left(\tilde{a}_{4}+1\right) \ln \left(\frac{1+\tilde{a}_{4}}{\tilde{a}_{4}}\right)\left(\tilde{m}^{2}-2 \tilde{g}^{2}+\frac{1-\tilde{a}_{4}}{2}\right)-4 \tilde{g}^{2} \sqrt{1+f^{2}}\right]
\end{aligned}
$$

in terms of the rescaled quantities $\tilde{m}=m \Lambda^{-1}, \tilde{a}=a \Lambda^{-2}, \tilde{g}^{2}=g^{2} f_{1} \Lambda^{-2}$, and $f=4 f_{2} / f_{1}$. Note that $f$ is a real-valued parameter. When we consider the limit of vanishing bare mass $m_{0}$, denote $\tilde{G}=G \Lambda^{2}$, and use the result for the momentum integral $I_{P T_{2}}$, the general gap equation (12) takes the algebraic form

$$
\begin{aligned}
\frac{2 \pi^{2}}{\tilde{G} N_{c} N_{f}}= & \frac{1}{8 \tilde{g}^{2} \sqrt{1+f^{2}}}\left\{4 \tilde{g}^{2} \sqrt{1+f^{2}}-\left(\tilde{a}_{1}+1\right) \ln \left(\frac{1+\tilde{a}_{1}}{\tilde{a}_{1}}\right)\left(\tilde{m}^{2}-2 \tilde{g}^{2}+\frac{1-\tilde{a}_{1}}{2}\right)\right. \\
& -\left(\tilde{a}_{2}+1\right) \ln \left(\frac{1+\tilde{a}_{2}}{\tilde{a}_{2}}\right)\left(\tilde{m}^{2}-2 \tilde{g}^{2}+\frac{1-\tilde{a}_{2}}{2}\right)+\left(\tilde{a}_{3}+1\right) \ln \left(\frac{1+\tilde{a}_{3}}{\tilde{a}_{3}}\right)\left(\tilde{m}^{2}-2 \tilde{g}^{2}+\frac{1-\tilde{a}_{3}}{2}\right) \\
& \left.+\left(\tilde{a}_{4}+1\right) \ln \left(\frac{1+\tilde{a}_{4}}{\tilde{a}_{4}}\right)\left(\tilde{m}^{2}-2 \tilde{g}^{2}+\frac{1-\tilde{a}_{4}}{2}\right)\right\}
\end{aligned}
$$

in the limit of vanishing bare mass $m_{0}$. We note that in the limit $\tilde{g} \rightarrow 0$ one recovers the known gap equation of the conventional Hermitian NJL model in this regularization scheme [11]:

$$
\frac{2 \pi^{2}}{\tilde{G} N_{c} N_{f}}=1-\tilde{m}^{2} \ln \left(\frac{1+\tilde{m}^{2}}{\tilde{m}^{2}}\right) .
$$

The behavior of the right-hand side of (33) is shown in Fig. 2(a) as a function of $\tilde{m}$ and for different values of $\tilde{g}$. The parameter $f=0.5$ is fixed, but is representative of the general behavior. The horizontal line denotes the constant real positive left-hand side of (33) for $\tilde{G}=3.93, N_{c}=3$, and $N_{f}=2$. For sufficiently small coupling values of $\tilde{g}$, an intersection, and therefore a real fermion mass solution $\tilde{m}$ to the gap equation, can always be found. However, beyond a critical value $\tilde{g}_{\text {crit }}$ the maximum of the right-hand side no longer exceeds the constant given by the left-hand side and real solutions no longer exist. The value of this critical coupling depends on the parameter $f$ and its behavior is shown in Fig. 2(b). It approaches zero asymptotically as $f$ increases to infinity. Figure 2(c) displays the behavior of the fermion mass solution $m$ (in $\mathrm{MeV}$ ) as a function of $\tilde{g}$ up to the corresponding critical couplings for different values of $f$. We note that, independent of $f$, the effective mass decreases with increasing coupling $\tilde{g}$. Thus, a current quark mass cannot be generated dynamically in this model.

Altogether, we find that - similar to the model discussed in Sec. III A-this $\mathcal{P} \mathcal{T}$-symmetric modified (nonHermitian) NJL model admits a finite region of unbroken $\mathcal{P T}$ symmetry, even for the vanishing bare mass $m_{0}$. However, while real fermion mass solutions do exist for coupling values up to the critical value $\tilde{g}_{\text {crit }}$, these solutions always describe an effective mass loss; an additional dynamical mass generation over and above that obtained through the four-fermion interaction is not possible.

\section{C. $\Gamma_{a P T_{1}}=i B_{\mu} \gamma^{\mu}$}

The case of the non- $\mathcal{P} \mathcal{T}$-symmetric model based on including the vector background field $\Gamma_{a P T_{1}}=i B_{\mu} \gamma^{\mu}$ is structurally very similar to the $\mathcal{P} \mathcal{T}$-symmetric one discussed in Sec. III A. It is also the only other non-Hermitian, 


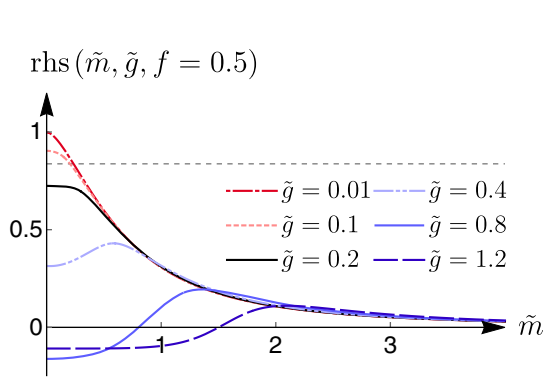

(a)

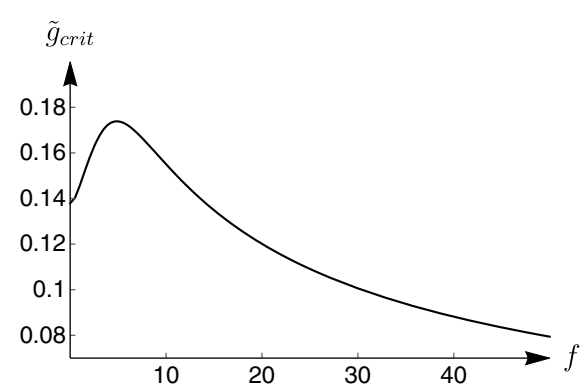

(b)

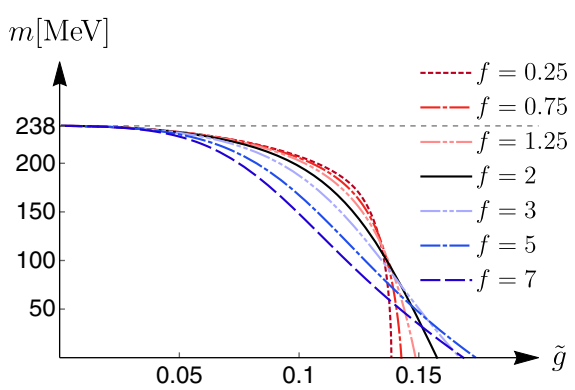

(c)

FIG. 2. (a) Behavior of the right-hand side of the gap equation (33) based on $\Gamma_{P T_{2}}$ as a function of the scaled mass $\tilde{m}=m / \Lambda$ and for different values of the coupling $\tilde{g}$. The value for $f=0.5$ is fixed, but this case is representative of the general behavior. The horizontal line represents the left-hand side of the gap equation. (b) Behavior of the critical coupling value $\tilde{g}_{\text {crit }}$ up to which the gap equation (33) has real mass solutions as a function of $f$. (c) Behavior of the effective fermion mass $m$ in MeV as a function of the coupling constant up to the corresponding critical values for different values of $f$.

bilinear extension of the NJL model that preserves chiral symmetry, as discussed in Sec. II.

To find the effective fermion mass of the model, we first rationalize the full propagator

$$
S(p)=\left(\not p-m-i g B_{\mu} \gamma^{\mu}\right)^{-1},
$$

finding that it can be written as

$$
S(p)=\frac{\not p+m-i B_{\mu} \gamma^{\mu}}{p^{2}-m^{2}-g^{2} B^{2}-2 i g B_{\mu} p^{\mu}},
$$

from which the spinor trace is readily obtained:

$$
\operatorname{tr}[S(p)]=\frac{4 m}{p^{2}-m^{2}-g^{2} B^{2}-2 i g B_{\mu} p^{\mu}} .
$$

Integrating out the four-momentum dependence of this expression results in an algebraic form of the gap equation of the model, cf. Eq. (13). As described previously in Sec. III A, we evaluate this integral in the Euclidean four-momentum cutoff regularization scheme: The cutoff
$\Lambda$ is introduced after transforming to coordinates $p_{0}=i p_{4}$ and $B_{0}=i B_{4}$, such that $p^{2}=-p_{E}^{2}, B^{2}=-B_{E}^{2}$, and $B_{\mu} p^{\mu}=-B_{E} \cdot p_{E}$. Thus,

$$
I_{a P T_{1}}=\int^{\Lambda} d^{4} p_{E} \frac{-4 i m}{p_{E}^{2}+m^{2}-g^{2} B_{E}^{2}-2 i g\left(B_{E} \cdot p_{E}\right)} .
$$

In spherical coordinates with the zenith direction along $B_{E}$, the Euclidean scalar product $B_{E} \cdot p_{E}=\left|B_{E}\right| r \cos \theta$ contains only the radius $r=\left|p_{E}\right|$ and the zenith angle $\theta$, so that we can write

$$
I_{a P T_{1}}=\frac{8 \pi m}{g\left|B_{E}\right|} \int_{0}^{\Lambda} d r r^{2} \int_{0}^{\pi} d \theta \frac{\sin ^{2} \theta}{A(r)+\cos \theta},
$$

where $A(r)=\left(r^{2}+m^{2}-g^{2}\left|B_{E}\right|^{2}\right) /\left(-2 i g r\left|B_{E}\right|\right)$. Both the angular and the resulting radial integrals are standard [18]. In terms of the rescaled quantities $\tilde{m}=m \Lambda^{-1}$ and $\tilde{g}=g\left|B_{E}\right| \Lambda^{-1}$, the latter of which is proportional to the amplitude of the vector background field, (37) becomes

$$
\begin{aligned}
I_{a P T_{1}}= & \frac{i \Lambda^{3} \pi^{2} \tilde{m}}{\tilde{g}^{2}}\left\{1+\left(\tilde{m}^{2}-\tilde{g}^{2}\right)\left(2+\tilde{m}^{2}+\tilde{g}^{2}\right)-\left(1+\tilde{m}^{2}+\tilde{g}^{2}\right) \sqrt{\left(1+\tilde{m}^{2}+\tilde{g}^{2}\right)^{2}-4 \tilde{g}^{2} \tilde{m}^{2}}\right. \\
& \left.+4 \tilde{m}^{2} \tilde{g}^{2} \ln \left[\frac{1}{2 \tilde{m}^{2}}\left(\sqrt{\left(1+\tilde{m}^{2}+\tilde{g}^{2}\right)^{2}-4 \tilde{g}^{2} \tilde{m}^{2}}+1+\tilde{m}^{2}+\tilde{g}^{2}\right)\right]\right\} .
\end{aligned}
$$

Denoting $\tilde{G}=G \Lambda^{2}$, the general gap equation (13) in the limit of vanishing bare mass $m_{0}$ thus takes the algebraic form

$$
\begin{aligned}
\frac{2 \pi^{2}}{\tilde{G} N_{c} N_{f}}= & \frac{1}{4 \tilde{g}^{2}}\left\{\left(1+\tilde{m}^{2}+\tilde{g}^{2}\right) \sqrt{\left(1+\tilde{m}^{2}+\tilde{g}^{2}\right)^{2}-4 \tilde{g}^{2} \tilde{m}^{2}}-1-\left(\tilde{m}^{2}-\tilde{g}^{2}\right)\left(2+\tilde{m}^{2}+\tilde{g}^{2}\right)\right. \\
& \left.-4 \tilde{m}^{2} \tilde{g}^{2} \ln \left[\frac{1}{2 \tilde{m}^{2}}\left(\sqrt{\left(1+\tilde{m}^{2}+\tilde{g}^{2}\right)^{2}-4 \tilde{g}^{2} \tilde{m}^{2}}+1+\tilde{m}^{2}+\tilde{g}^{2}\right)\right]\right\} .
\end{aligned}
$$




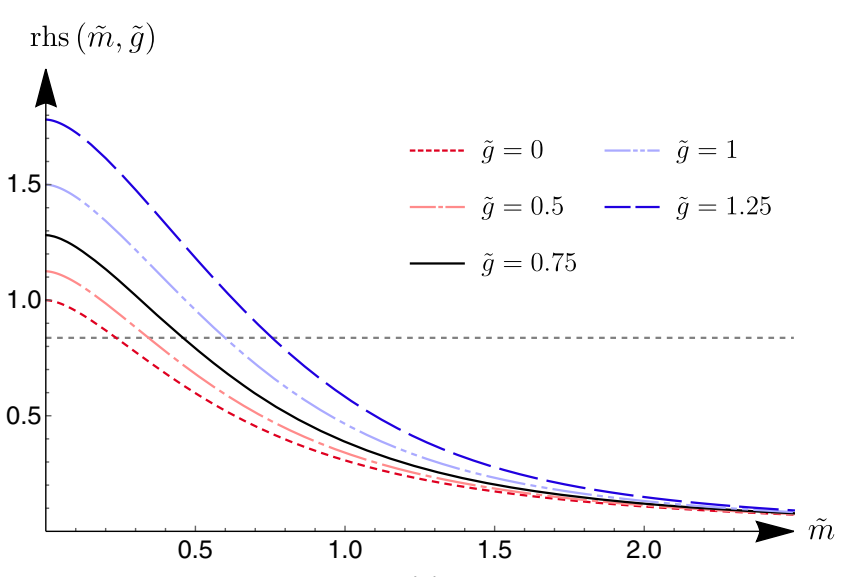

(a)

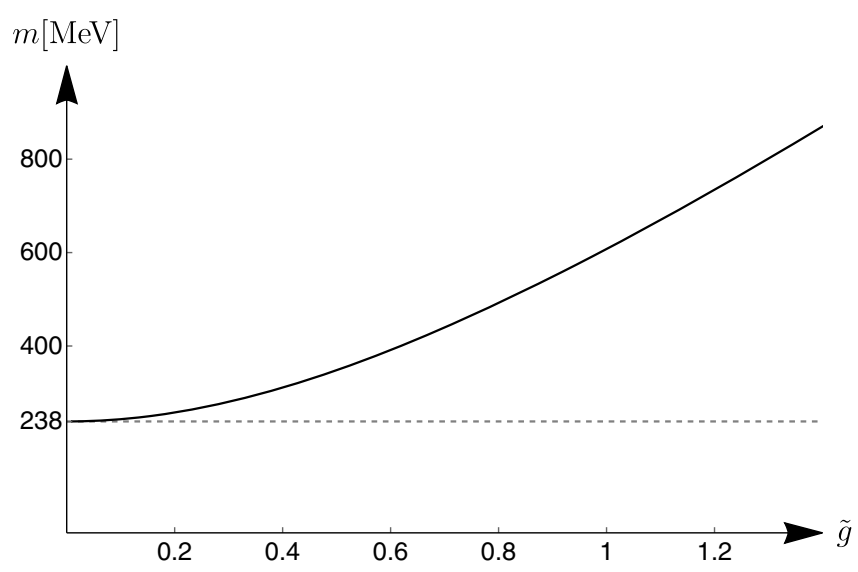

(b)

FIG. 3. In (a) the behavior of the right-hand side of the gap equation (39) based on $\Gamma_{a P T_{1}}$ is shown as a function of the mass $\tilde{m}$ for different values of the coupling $\tilde{g}$. The horizontal line represents the left-hand side of the gap equation. Figure (b) displays the behavior of the real mass solutions $m$ in $\mathrm{MeV}$ as function of the coupling constant.

The gap equation (34) of the standard NJL model is recovered in the limit $\tilde{g} \rightarrow 0$.

The right-hand side of (39) is a real function of the scaled mass $\tilde{m}$ and coupling constant $\tilde{g}$. Its behavior is shown in Fig. 3(a). Intersections with the real constant left-and side, visualized by a horizontal line, can always be found when evaluated within the context of QCD. The corresponding fermion mass solution $m$ (in $\mathrm{MeV}$ ) is shown in Fig. 3(b) as a function of the coupling constant $\tilde{g}$. Note that, contrary to the $\mathcal{P} \mathcal{T}$-symmetric models discussed in III A and III B, real mass solutions are not limited to a finite coupling constant regime and increase monotonously with the value of the coupling constant. A current quark mass is generated dynamically in this model: the equivalent of a bare up quark mass, $m_{u}=(1.7-3.3) \mathrm{MeV}$, requires coupling values in the range $\tilde{g} \approx(0.059-0.083)$ and for the bare down quark mass, $\quad m_{d}=(4.1-5.8) \mathrm{MeV}$, coupling values $\tilde{g} \approx$ (0.092-0.110) are necessary.

Altogether, we find that real fermion mass solutions are obtained for all values of the coupling constant $\tilde{g}$ in this chirally symmetric non-Hermitian extension of the NJL model, even though $\mathcal{P} \mathcal{T}$ symmetry is broken explicitly. Moreover, the generation of an additional dynamical mass mimicking a bare fermion mass occurs.

\section{D. $\Gamma_{a P T_{2}}=\gamma_{5}$}

The analysis of the non- $\mathcal{P} \mathcal{T}$-symmetric model based on the pseudoscalar term $\Gamma_{a P T_{2}}=\gamma_{5}$ is straightforward: The full fermion propagator

$$
S(p)=\left(\not p-m-g \gamma_{5}\right)^{-1}
$$

can be rationalized and simplified to the form

$$
S(p)=\frac{\not p+m-g \gamma_{5}}{p^{2}-m^{2}+g^{2}},
$$

which has the spinor trace

$$
\operatorname{tr}[S(p)]=\frac{4 m}{\left(p^{2}-m^{2}+g^{2}\right)} .
$$

Since this function only depends on the square of the four-momentum, the momentum integral (13) can be evaluated directly: in spherical Euclidean coordinates, with $p^{2}=-p_{E}^{2}$, we introduce the Euclidean four-momentum cutoff $\Lambda$ as the bound of the radial integration and subsequently find that

$I_{a P T_{2}}=4 i \Lambda^{3} \pi^{2} \tilde{m}\left[\left(\tilde{m}^{2}-\tilde{g}^{2}\right) \ln \left(\frac{1+\tilde{m}^{2}-\tilde{g}^{2}}{\tilde{m}^{2}-\tilde{g}^{2}}\right)-1\right]$,

in terms of the rescaled parameters $\tilde{m}=m \Lambda^{-1}$ and $\tilde{g}=g \Lambda^{-1}$. The gap equation (12) of this model thus takes the algebraic form

$$
\frac{2 \pi^{2}}{\tilde{G} N_{c} N_{f}}=1-\left(\tilde{m}^{2}-\tilde{g}^{2}\right) \ln \left(\frac{1+\tilde{m}^{2}-\tilde{g}^{2}}{\tilde{m}^{2}-\tilde{g}^{2}}\right)
$$

in the limit $m_{0} \rightarrow 0$, which reduces to the gap equation (34) of the standard NJL model in the limit of vanishing coupling constant values $\tilde{g}$.

Equation (43) can be solved algebraically in terms of the Lambert $W$ function [19]. We find the real fermion mass solution

$$
\tilde{m}=\sqrt{\tilde{g}^{2}+\left[\frac{1}{c} W_{-1}\left(c e^{c}\right)-1\right]^{-1}}=\sqrt{\tilde{g}^{2}+\tilde{m}_{\mathrm{NJL}}^{2}},
$$




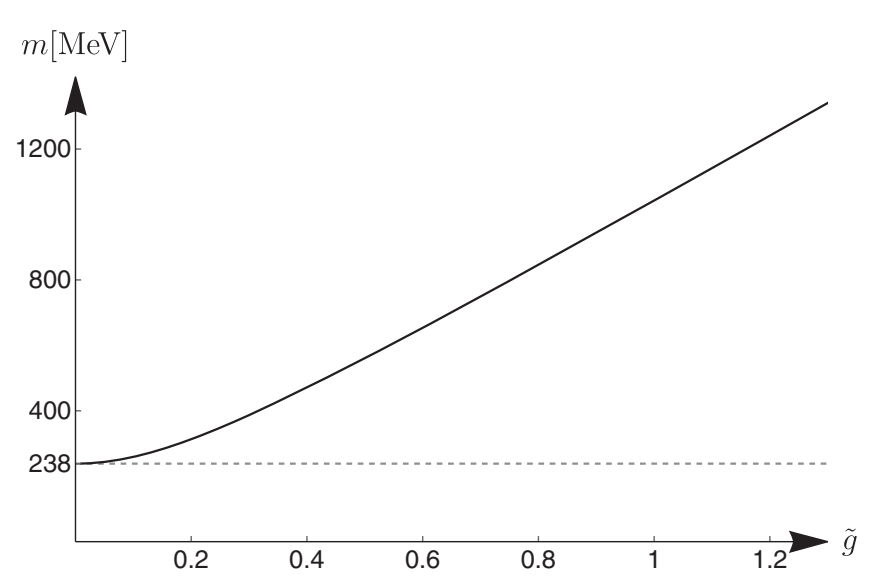

FIG. 4. Behavior of the fermion mass solution $m$ of the modified NJL model based on $\Gamma_{a P T_{2}}$ as a function of the scaled coupling constant $\tilde{g}=g \Lambda^{-1}$.

where, in the context of QCD, $c=2 \pi^{2} / \tilde{G} N_{c} N_{f}-1$ is a negative real constant and $\tilde{m}_{\mathrm{NJL}}$ is the (scaled) fermion mass of the standard NJL model. The behavior of this solution is shown in Fig. 4. Similar to the case discussed in Sec. III C, we find that the existence of real fermion mass solutions is, in contrast to the $\mathcal{P} \mathcal{T}$-symmetric models, not limited to a finite coupling constant regime and the mass increases again monotonously with the coupling constant value $\tilde{g}$. A current quark mass equivalent to that of a bare up quark mass, $m_{u}=(1.7-3.3) \mathrm{MeV}$, is dynamically generated for coupling values in the range $\tilde{g} \approx(0.028-0.039)$ and for the equivalent of a the bare down quark mass, $m_{d}=(4.1-5.8) \mathrm{MeV}$, coupling values $\tilde{g} \approx(0.044-0.052)$ are required.

We remark that the analytical mass solution (44) coincides with that found for the renormalized, modified GrossNeveu model in [5], which can be seen as a $1+1$ dimensional version of the NJL model. We emphasize that while the pseudoscalar term $\Gamma_{a P T_{2}}=\gamma_{5}$ does not give rise to a $\mathcal{P} \mathcal{T}$-symmetric bilinear in $3+1$ dimensions, its $1+1$ dimensional equivalent is, in fact, the only possible $\mathcal{P} \mathcal{T}$-symmetric, non-Hermitian, bilinear extension of the Gross-Neveu model [4,5].

Altogether, we find that the fermion mass solution of this model resembles that found in Sec. III C: even though $\mathcal{P} \mathcal{T}$ symmetry is explicitly broken, real mass solutions are found for all coupling constant values and an additional dynamical mass generation, over and above that generated by the standard NJL model, occurs. In contrast to the model based on $\Gamma_{a P T_{1}}$, this model does not, however, preserve chiral symmetry.

\section{E. $\Gamma_{a P T_{3}}=i \rrbracket$}

Modifying the NJL model by adding a bilinear term based on the scalar $\Gamma_{a P T_{3}}=i \rrbracket$ amounts to including an imaginary mass contribution proportional to the coupling constant $g$ into the propagator:

$$
S(p)=(\not p-m-i g)^{-1} .
$$

Correspondingly, the gap equation (12) is obtained as that of the standard NJL model with an additional contribution to the mass terms arising in the momentum integral on the right-hand side. In the four-momentum cutoff regularization scheme it reads

$$
\frac{2 \pi^{2}}{\tilde{G} N_{c} N_{f}}=\frac{\tilde{m}+i \tilde{g}}{\tilde{m}}\left\{1-(\tilde{m}+i \tilde{g})^{2} \ln \left[\frac{1+(\tilde{m}+i \tilde{g})^{2}}{(\tilde{m}+i \tilde{g})^{2}}\right]\right\}
$$

in terms of the rescaled parameters $\tilde{m}=m \Lambda^{-1}$ and $\tilde{g}=$ $g \Lambda^{-1}$. For a vanishing coupling constant $\tilde{g}$, this simplifies to the gap equation (34) of the standard NJL model in this regularization scheme. Otherwise, the right-hand side of (45) takes on inherently complex values and an intersection with the purely real left-hand side, and thus a real fermion mass solution cannot be found.

The modified NJL model based on $\Gamma_{a P T_{3}}$ is thus the only one of the non-Hermitian systems considered here that does not admit real fermionic mass solutions at all.

In summary: For $\mathcal{P} \mathcal{T}$-symmetric theories based on $\Gamma_{P T_{1}}$ and $\Gamma_{P T_{2}}$ that symmetry was realized exclusively as a broken symmetry phase in the underlying modified free Dirac models, generally giving rise to complex fermion masses [4]. However, the addition of two-body interactions via the associated modified NJL models restores this symmetry, leading to a finite region where $\mathcal{P} \mathcal{T}$ is unbroken. This is manifested through the real fermion mass solutions found in both cases (cf. III A and III B). Nevertheless, an additional dynamical mass generation is only possible in the model extended by a pseudovector background field $\Gamma_{P T_{1}}$ (III A), and in this case only for specific values of the coupling strength $\tilde{g}$.

In contrast, it is surprising that one can find real fermion masses in the non-Hermitian non- $\mathcal{P} \mathcal{T}$-symmetric models that include $\Gamma_{a P T_{1}}$ and $\Gamma_{a P T_{2}}$ at all, cf. III C and III $\mathrm{D}$, as these cannot be attributed to an unbroken symmetry phase of any kind. Furthermore, in both models these real mass solutions are not restricted to finite coupling regions, but are obtained for all possible values of the coupling constant $\tilde{g}$. In addition, these solutions always allow for increasing dynamically generated masses, over and above those obtained from the standard NJL model. In the model containing $\Gamma_{a P T_{3}}$ in III E no real fermion masses were found.

Thus, the effect of a small bare mass $m_{0}$ in the standard NJL model can be mimicked by including any of the three non-Hermitian bilinear terms $\Gamma_{P T_{1}}, \Gamma_{a P T_{1}}$, or $\Gamma_{a P T_{2}}$ into the NJL model. 


\section{MESON MASSES}

We have identified four non-Hermitian bilinear extensions of the NJL model that give rise to purely real fermion mass solutions, three of which allow for an additional dynamical generation of fermion mass as a function of the coupling strength $\tilde{g}$. In this section we investigate the masses of the corresponding scalar and pseudoscalar bound states for these three models. In the context of QCD these correspond to the $\sigma$ and $\pi$ mesons, respectively. First we recall the established self-consistent mass equations for these bound states and how they can be obtained from the effective meson interaction, cf. $[9,11,16]$. In Sec. IV A we call to mind the resulting meson mass solutions for the standard NJL model, followed by a discussion of the results found for the modified NJL model based on including the pseudoscalar term $\Gamma_{a P T_{2}}=\gamma_{5}$, in which both chiral and $\mathcal{P} \mathcal{T}$ symmetry are broken explicitly, cf. Sec. IV B. In Secs. IV C and IV D, the scalar and pseudoscalar meson masses are analyzed for the modified NJL models based on the vector background field $\Gamma_{a P T_{1}}=i B_{\mu} \gamma^{\mu}$ and the pseudovector background field $\Gamma_{P T_{1}}=i \gamma_{5} B_{\mu} \gamma^{\mu}$, both of which are chirally symmetric.

Following the discussions in [11,16] for the standard NJL model, one must construct the effective meson interaction $U_{\alpha \alpha^{\prime}, \beta \beta^{\prime}}$, where all relevant degrees of freedom are subsumed in the indices $\alpha, \alpha^{\prime}, \beta, \beta^{\prime}$. The effective interaction is expressed in terms of the (proper) polarization insertion $\Pi_{\lambda \lambda^{\prime}, \mu \mu^{\prime}}$ :

$$
\begin{aligned}
i U_{\alpha \alpha^{\prime}, \beta \beta^{\prime}}(k)= & i U_{\alpha \alpha^{\prime}, \beta \beta^{\prime}}^{0}(k) \\
& +i U_{\alpha \alpha^{\prime}, \lambda \lambda^{\prime}}^{0}(k)\left[-i \Pi_{\lambda \lambda^{\prime}, \mu \mu^{\prime}}(k)\right] i U_{\mu \mu^{\prime}, \beta \beta^{\prime}}(k) .
\end{aligned}
$$

Here $U_{\alpha \alpha^{\prime}, \beta \beta^{\prime}}^{0}$ denotes the bare four-point interaction of the model, which (in position space) has the form

$$
\begin{aligned}
U_{\alpha \alpha^{\prime}, \beta \beta^{\prime}}^{0}(x, y)= & 2 G \delta^{(4)}(x-y) \\
& \times\left[\delta_{\alpha \alpha^{\prime}} \delta_{\beta \beta^{\prime}}+\left(i \gamma_{5} \vec{\tau}\right)_{\alpha \alpha^{\prime}}\left(i \gamma_{5} \vec{\tau}\right)_{\beta \beta^{\prime}}\right] .
\end{aligned}
$$

Choosing $\quad U_{\alpha \alpha^{\prime}, \beta \beta^{\prime}}^{0}(k)=U^{0}(k) \delta_{\alpha \alpha^{\prime}} \delta_{\beta \beta^{\prime}} \quad$ or $\quad U_{\alpha \alpha^{\prime}, \beta \beta^{\prime}}^{0}(k)=$ $U^{0}(k)\left(i \gamma_{5} \vec{\tau}\right)_{\alpha \alpha^{\prime}}\left(i \gamma_{5} \vec{\tau}\right)_{\beta \beta^{\prime}}$ (46) simplifies to

$$
i U(k)=i U^{0}(k)+i U^{0}(k)\left[-i \Pi^{s / p s}(k)\right] i U(k),
$$

where $\Pi^{s}(k)=\delta_{\lambda \lambda^{\prime}} \Pi_{\lambda \lambda^{\prime}, \mu \mu^{\prime}}^{s}(k) \delta_{\mu \mu^{\prime}}$ and $\Pi^{p s}(k)=\left(i \gamma_{5} \vec{\tau}\right)_{\lambda \lambda^{\prime}} \times$ $\Pi_{\lambda \lambda^{\prime}, \mu \mu^{\prime}}^{p s}(k)\left(i \gamma_{5} \vec{\tau}\right)_{\mu \mu^{\prime}}$ for the scalar and pseudoscalar cases, respectively. Equation (48) is a geometric progression that can be summed to the form

$$
i U(k)=\frac{i U^{0}(k)}{1-2 G \Pi^{s / p s}(k)} .
$$

The pole of this expression corresponds to that of the general scalar or pseudoscalar mode propagator, that is, it occurs when $k^{2}=m_{s / p s}^{2}$. Thus, in order to determine the mass $m_{s / p s}$, we have to solve the equation

$$
1-2 G \Pi^{s / p s}(k)=0 .
$$

The lowest order polarization insertion is a closed fermion loop. One can improve this approximation by implementing self-consistency, i.e., we replace the free fermion propagator $S^{0}$ by the full propagator $S$. Evaluating this contribution with the corresponding vertex functions gives

$$
-i \Pi^{s}(k)=N_{c} N_{f} \int \frac{d^{4} p}{(2 \pi)^{4}} \operatorname{tr}[S(p+k) S(p)]
$$

and similarly

$-i \Pi^{p s}(k)=-N_{c} N_{f} \int \frac{d^{4} p}{(2 \pi)^{4}} \operatorname{tr}\left[\gamma_{5} S(p+k) \gamma_{5} S(p)\right]$

for each pseudoscalar channel.

Equation (49) at $k^{2}=m_{s / p s}^{2}$, together with (50) and (51), thus determines the mass of the scalar and pseudoscalar bound states. To simplify notation, we use the general form of the gap equation (12), as discussed in Sec. III, in the limit of vanishing bare mass $m_{0}$, and rewrite Eq. (49) as

$$
R^{s / p s}(p, k)-I / m=0 \quad \text { at } k^{2}=m_{s / p s}^{2},
$$

where

$$
\begin{gathered}
R^{s}(k)=\int d^{4} p \operatorname{tr}[S(p+k) S(p)], \\
R^{p s}(k)=-\int d^{4} p \operatorname{tr}\left[\gamma_{5} S(p+k) \gamma_{5} S(p)\right],
\end{gathered}
$$

and $I$ defined in (13) is evaluated through

$$
\frac{I}{m}=\frac{1}{2 m} \int d^{4} p(\operatorname{tr}[S(p)]+\operatorname{tr}[S(p+k)]) .
$$

As was the case for the gap equation, the equation for the self-consistent meson masses (52) of the standard NJL model retains the same general structure for the modified NJL models constructed with the Hamiltonian (2), since the influence of the additional bilinear terms is accounted for in the full fermion propagator $S(p)$, cf. [5]. Similar to the discussion of the gap equation in Sec. III, our analysis of the meson masses thus relies on the evaluation of spinor traces and momentum integrations in (53) and (54) involving the appropriate fermion propagators of the modified NJL models. 


\section{A. Standard NJL model}

In the standard NJL model, the evaluation of the selfconsistent meson mass equation (52) is based on the full Dirac fermion propagator

$$
S(p)=(\not p-m)^{-1}=\frac{\not p+m}{p^{2}-m^{2}},
$$

with $m=m_{\mathrm{NJL}}$ being the effective fermion mass determined by the gap equation (12) in the chiral limit. From this, the spinor traces in Eqs. (53)-(55) are readily calculated, leading to the expressions

$$
R^{s}(k)=\int d^{4} p \frac{4\left(p^{2}+p_{\mu} k^{\mu}+m^{2}\right)}{\left((p+k)^{2}-m^{2}\right)\left(p^{2}-m^{2}\right)}
$$

and

$$
R^{p s}(k)=\int d^{4} p \frac{4\left(p^{2}+p_{\mu} k^{\mu}-m^{2}\right)}{\left((p+k)^{2}-m^{2}\right)\left(p^{2}-m^{2}\right)},
$$

as well as

$$
\frac{I}{m}=\int d^{4} p \frac{4\left(p^{2}+p_{\mu} k^{\mu}-m^{2}\right)+2 k^{2}}{\left((p+k)^{2}-m^{2}\right)\left(p^{2}-m^{2}\right)} .
$$

Equation (52) then takes the form of the well-known conditions

$$
\begin{array}{r}
0=\left(k^{2}-4 m^{2}\right) \int d^{4} p \frac{1}{\left((p+k)^{2}-m^{2}\right)\left(p^{2}-m^{2}\right)}, \\
0=k^{2} \int d^{4} p \frac{1}{\left((p+k)^{2}-m^{2}\right)\left(p^{2}-m^{2}\right)}
\end{array}
$$

at $k^{2}=m_{s / p s}^{2}$ for the scalar and pseudoscalar meson masses respectively, cf. [11].

These conditions give rise to the apparent solutions that $m_{s}^{2}=4 m^{2}=4 m_{\mathrm{NJL}}^{2}$ and $m_{p s}^{2}=0$, the latter of which is the Nambu-Goldstone mode in this model, in which chiral symmetry is spontaneously broken. In addition, a simultaneous solution of $m_{s}$ and $m_{p s}$ arises when the integral in (56) and (57) vanishes. In the Euclidean fourmomentum cutoff regularization scheme, this happens at $|k|=m_{s / p s} \approx 0.712 \Lambda$. The mass spectrum of the scalar and pseudoscalar modes in the NJL model thus not only contains the Nambu-Goldstone mode and its chiral partner, but also an additional finite mass solution, which describes a scalar/pseudoscalar mode degeneracy.

\section{B. $\Gamma_{a P T_{2}}=\gamma_{5}$}

When the NJL model is modified by the pseudoscalar term $\Gamma_{a P T_{2}}=\gamma_{5}$, the resulting extended model is neither Hermitian, nor $\mathcal{P} \mathcal{T}$-symmetric, nor chirally symmetric. Nevertheless, the model gives rise to real fermion masses as was shown in Sec. III D. Looking now at the selfconsistent mass equation (52) for the mesonic bound states, we find that evaluating the spinor traces in (53) to (55) for the fermion propagator associated with this model and given in (40), yields

$$
R_{a P T_{2}}^{s}(k)=\int d^{4} p \frac{4\left(p^{2}+p_{\mu} k^{\mu}+m^{2}+g^{2}\right)}{\left((p+k)^{2}-m^{2}+g^{2}\right)\left(p^{2}-m^{2}+g^{2}\right)},
$$

$$
R_{a P T_{2}}^{p s}(k)=\int d^{4} p \frac{4\left(p^{2}+p_{\mu} k^{\mu}-m^{2}-g^{2}\right)}{\left((p+k)^{2}-m^{2}+g^{2}\right)\left(p^{2}-m^{2}+g^{2}\right)},
$$

$$
\frac{I_{a P T_{2}}}{m}=\int d^{4} p \frac{4\left(p^{2}+p_{\mu} k^{\mu}-m^{2}+g^{2}\right)+2 k^{2}}{\left((p+k)^{2}-m^{2}+g^{2}\right)\left(p^{2}-m^{2}+g^{2}\right)} .
$$

The condition (52) for the scalar and pseudoscalar meson modes thus takes the form

$$
0=\left(k^{2}-4 m^{2}\right) \int d^{4} p \frac{1}{\left((p+k)^{2}-m^{2}+g^{2}\right)\left(p^{2}-m^{2}+g^{2}\right)},
$$

$$
0=\left(k^{2}+4 g^{2}\right) \int d^{4} p \frac{1}{\left((p+k)^{2}-m^{2}+g^{2}\right)\left(p^{2}-m^{2}+g^{2}\right)},
$$

respectively, at $k^{2}=m_{s / p s}^{2}$. This closely resembles the conditions (56) and (57) of the standard NJL model.

We note, that (unsurprisingly) the appearance of a Nambu-Goldstone mode in the standard NJL model breaks down with the inclusion of the chiral symmetry breaking term $\Gamma_{a P T_{2}}$. In its place we find the apparent solution to (62) that $m_{p s}^{2}=-4 g^{2}$, i.e., the pseudoscalar solution is a tachyonic state with mass $m_{p s}= \pm 2 i g$. The apparent solution to (61) for the scalar meson, however, remains structurally identical to that of the standard NJL model, cf. (56); but the effective fermion mass $m$ is now a function of the coupling constant $g$ in this modified model; see (44). Thus, $m_{s}^{2}=4 m^{2}=4\left(m_{\mathrm{NJL}}^{2}+g^{2}\right)$. The dynamical generation of a fermion mass thus also affects the scalar meson. In addition, the dependence of the fermion mass on the coupling constant $g$ also affects the appearance of a simultaneous solution of $m_{s}$ and $m_{p s}$ when the momentum integral in (61) and (62) vanishes. In fact, it counteracts the explicit $g$ dependence of the momentum integrals, leaving the same expression that is found in the standard NJL model, cf. (56) and (57). We thus find a scalar/pseudoscalar mode degeneracy with the same mass, $m_{s / p s} \approx \pm 0.712 \Lambda$ in the Euclidean four-momentum cutoff regularization, which 
is unaffected by the explicit breaking of chiral symmetry in this model.

Altogether, the bilinear based on $\Gamma_{a P T_{2}}$, while causing a dynamical mass gain of the fermion and the scalar meson, seems to act as a tachyonic instability for the pseudoscalar meson. The scalar/pseudoscalar mode degeneracy of the standard NJL model remains unchanged by this extension. We point out that, while the Nambu-Goldstone mode of the standard NJL model becomes tachyonic in this extended model, the combination of the nondegenerate scalar and pseudoscalar meson masses $m_{s}^{2}+m_{p s}^{2}=$ $4 m_{\mathrm{NJL}}^{2}$ remains valid.

$$
\text { C. } \Gamma_{a P T_{1}}=i B_{\mu} \gamma^{\mu}
$$

Having analyzed the modified NJL model, which breaks chiral symmetry explicitly, we now turn to the remaining two models in which chiral symmetry is conserved: the non-Hermitian models based on the vector background field $\Gamma_{a P T_{1}}=i B_{\mu} \gamma^{\mu}$, which we discuss in this section, and the pseudovector background field $\Gamma_{P T_{1}}=i \gamma_{5} B_{\mu} \gamma^{\mu}$, which is discussed in Sec. IV D. As per the results of Sec. III C, real fermion masses are dynamically generated for all values of the coupling constant in the former, non- $\mathcal{P} \mathcal{T}$ symmetric case. In the latter, $\mathcal{P} \mathcal{T}$-symmetric case, this happens in the finite region of coupling values smaller than $\tilde{g}_{d y n} \approx 1.183$, cf. Sec. III A.

For the NJL model that is extended by an anti-P $\mathcal{T}$ symmetric scalar background field term $\Gamma_{a P T_{1}}=i B_{\mu} \gamma^{\mu}$, calculating the spinor traces in the terms (53) and (54), with the fermion propagator as given in (35), results in the expressions

$$
R_{a P T_{1}}^{s}(k)=\int d^{4} p \frac{4\left(p^{2}+p_{\mu} k^{\mu}+m^{2}-g^{2} B^{2}-2 i g B_{\mu} p^{\mu}-i g B_{\mu} k^{\mu}\right)}{\left((p+k)^{2}-m^{2}-g^{2} B^{2}-2 i g B_{\mu}(p+k)^{\mu}\right)\left(p^{2}-m^{2}-g^{2} B^{2}-2 i g B_{\mu} p^{\mu}\right)}
$$

and

$$
R_{a P T_{1}}^{p s}(k)=\int d^{4} p \frac{4\left(p^{2}+p_{\mu} k^{\mu}-m^{2}-g^{2} B^{2}-2 i g B_{\mu} p^{\mu}-i g B_{\mu} k^{\mu}\right)}{\left((p+k)^{2}-m^{2}-g^{2} B^{2}-2 i g B_{\mu}(p+k)^{\mu}\right)\left(p^{2}-m^{2}-g^{2} B^{2}-2 i g B_{\mu} p^{\mu}\right)} .
$$

With the integral $I_{a P T_{1}}$ in the form (55) being

$$
\frac{I_{a P T_{1}}}{m}=\int d^{4} p \frac{4\left(p^{2}+p_{\mu} k^{\mu}-m^{2}-g^{2} B^{2}-2 i g B_{\mu} p^{\mu}-i g B_{\mu} k^{\mu}\right)+2 k^{2}}{\left((p+k)^{2}-m^{2}-g^{2} B^{2}-2 i g B_{\mu}(p+k)^{\mu}\right)\left(p^{2}-m^{2}-g^{2} B^{2}-2 i g B_{\mu} p^{\mu}\right)},
$$

we obtain the following form for the self-consistent mass equation (52) for the scalar and pseudoscalar modes:

$$
\begin{gathered}
0=\left(k^{2}-4 m^{2}\right) \int d^{4} p \frac{1}{\left((p+k)^{2}-m^{2}-g^{2} B^{2}-2 i g B_{\mu}(p+k)^{\mu}\right)\left(p^{2}-m^{2}-g^{2} B^{2}-2 i g B_{\mu} p^{\mu}\right)}, \\
0=k^{2} \int d^{4} p \frac{1}{\left((p+k)^{2}-m^{2}-g^{2} B^{2}-2 i g B_{\mu}(p+k)^{\mu}\right)\left(p^{2}-m^{2}-g^{2} B^{2}-2 i g B_{\mu} p^{\mu}\right)} .
\end{gathered}
$$

Both of the apparent solutions $m_{p s}^{2}=0$ and $m_{s}^{2}=4 m^{2}$ are structurally identical to those of the standard NJL model, cf. Eqs. (56) and (57). The existence of a massless Nambu-Goldstone mode remains unaffected throughout the extension of the model by the non-Hermitian anti- $\mathcal{P} \mathcal{T}$ symmetric term $\Gamma_{a P T_{1}}$. One can understand this, as chiral symmetry continues to be broken spontaneously.

The mass of the scalar meson, $m_{s}=2 m$, remains structurally identical as well, although once again the fermion mass $m$ now depends on the coupling constant $g$, cf. Sec. III C. The dynamical mass generation of the fermion is thus reflected in the mass of the scalar meson.
Furthermore, the appearance of the identical fourmomentum integrals in both conditions (63) and (64) suggests that an additional simultaneous solution of $m_{p s}$ and $m_{s}$ can be found here as well. However, the appearance of a term $B_{\mu} k^{\mu}$ in the denominator of this integral indicates that this solution depends on the angle between the background field $B_{\mu}$ and the meson momentum $k_{\mu}$. The roots of the momentum integral can be determined numerically in the Euclidean four-momentum cutoff regularization scheme for various values of the angle $\alpha$ between the Euclidean background field $B_{E}$ (where $B_{0}=i B_{4}$ ) and the Euclidean meson momentum $k_{E}$ (where $\left.k_{0}=i k_{4}\right)$. 


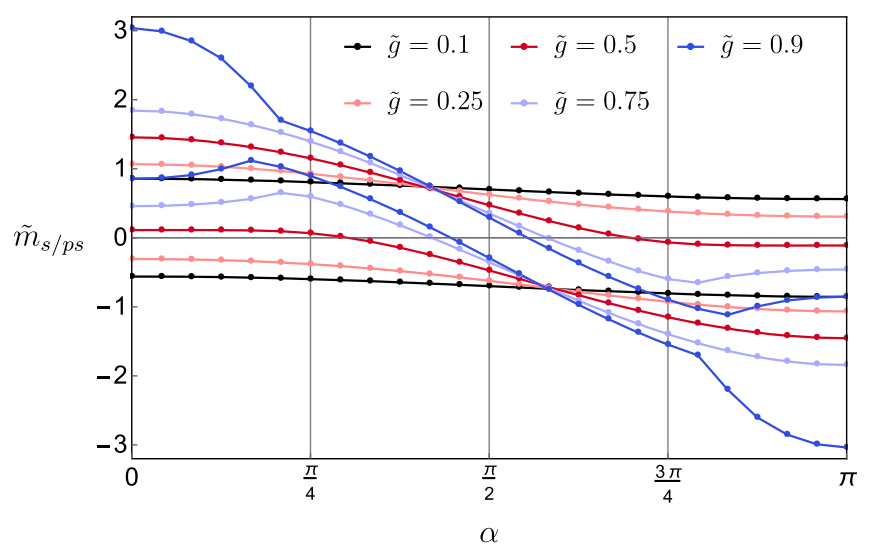

(a)

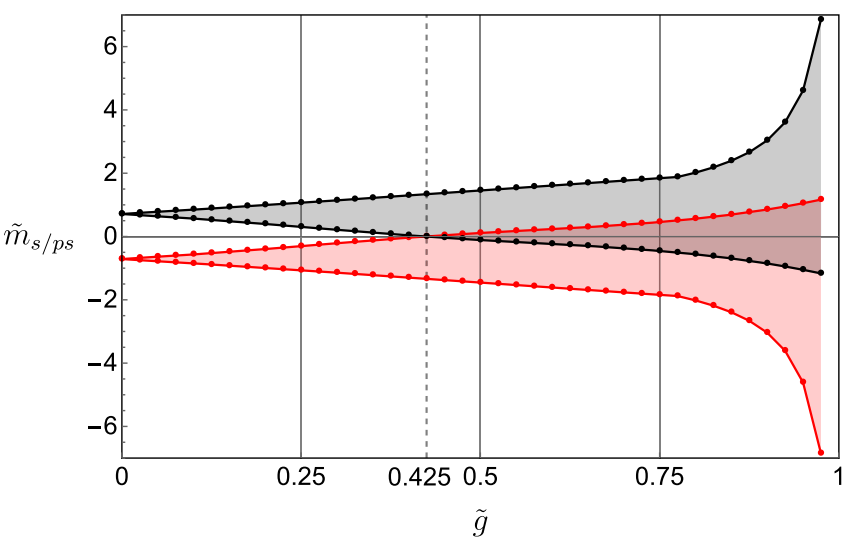

(b)

FIG. 5. In (a) the scaled mass of the scalar/pseudoscalar mode degeneracy $\tilde{m}_{s / p s}=m_{s / p s} \Lambda^{-1}$ in the modified NJL model based on $\Gamma_{a P T_{1}}$ is shown as function of the angle $\alpha$ between the Euclidean background field and the Euclidean meson momentum for fixed values of the coupling constant $\tilde{g}$. The region of accessible mass over this angle interval is visualized as function of $\tilde{g}$ in (b).

Figure 5(a) shows the behavior of the scaled degenerate meson mass $\tilde{m}_{s / p s}=m_{s / p s} \Lambda^{-1}$ as a function of the angle $\alpha$ for small values of the scaled coupling constant $\tilde{g}=$ $g\left|B_{E}\right| \Lambda^{-1}<1$, which describes the amplitude of the vector background field. In Fig. 5(b) the extremal values of the meson mass in the angular interval are visualized as a function of the coupling $\tilde{g}$ in the same region. Since the meson mass is a continuous function in $\alpha$, all mass values between these extrema can be found for some value of $\alpha$. This is shown in Fig. 5(b) as shaded regions. We note that for coupling values larger than $\tilde{g} \approx 0.425$, arbitrarily small (or vanishing) meson masses $m_{p s}$ can be realized.

Overall, we find a Nambu-Goldstone mode and a scalar meson with mass $m_{s}=2 m$ reflecting the dynamical mass generation of the fermion, plus the existence of an additional scalar/pseudoscalar degeneracy in the modified NJL model based on $\Gamma_{a P T_{1}}=i B_{\mu} \gamma^{\mu}$. The simultaneous solution shows an intricate dependence on the amplitude of the scalar background field and its angle relative to the meson momentum. For sufficiently large values of the coupling this mass solution may be arbitrarily small (or vanish entirely), but such values of the coupling also generate a significant fermion mass in the model.

\section{D. $\Gamma_{P T_{1}}=i \gamma_{5} B_{\mu} \gamma^{\mu}$}

When the standard NJL model is extended by a $\mathcal{P} \mathcal{T}$ symmetric pseudovector background field $\Gamma_{P T_{1}}=i \gamma_{5} B_{\mu} \gamma^{\mu}$, its rationalized fermion propagator is given by Eq. (15), which does not simplify as much as in the models discussed previously. The evaluation of the spinor traces in the terms (53), (54), and (55) thus yields somewhat cumbersome results. However, when inserted into the self-consistent meson mass equation (52) some contributions simplify and the conditions for the scalar and pseudoscalar meson masses become

$0=\int d^{4} p \frac{\left(k^{2}-4 m^{2}\right) A(p, k)+8 g^{2} B(p, k)}{\left[\left((p+k)^{2}-m^{2}-g^{2} B^{2}\right)^{2}-4 g^{2} m^{2} B^{2}+4 g^{2}\left(B_{\mu}(p+k)^{\mu}\right)^{2}\right]\left[\left(p^{2}-m^{2}-g^{2} B^{2}\right)^{2}-4 g^{2} m^{2} B^{2}+4 g^{2}\left(B_{\mu} p^{\mu}\right)^{2}\right]}$

and

$0=\int d^{4} p \frac{k^{2} A(p, k)+8 g^{2} B(p, k)}{\left[\left((p+k)^{2}-m^{2}-g^{2} B^{2}\right)^{2}-4 g^{2} m^{2} B^{2}+4 g^{2}\left(B_{\mu}(p+k)^{\mu}\right)^{2}\right]\left[\left(p^{2}-m^{2}-g^{2} B^{2}\right)^{2}-4 g^{2} m^{2} B^{2}+4 g^{2}\left(B_{\mu} p^{\mu}\right)^{2}\right]}$

respectively, at $k^{2}=m_{s / p s}^{2}$, where

$$
\begin{gathered}
A(p, k)=\left((p+k)^{2}-m^{2}+g^{2} B^{2}\right)\left(p^{2}-m^{2}+g^{2} B^{2}\right)+4 g^{2}\left[B^{2} p_{\mu}(p+k)^{\mu}-\left(B_{\mu} p^{\mu}\right)\left(B_{\nu}(p+k)^{\nu}\right)\right], \\
B(p, k)=2\left(p_{\mu} k^{\mu}\right)\left(B_{\nu} k^{\nu}\right)\left(B_{\alpha} p^{\alpha}\right)-B^{2}\left(p_{\mu} k^{\mu}\right)^{2}+p^{2} k^{2} B^{2}-k^{2}\left(B_{\mu} p^{\mu}\right)^{2}-p^{2}\left(B_{\mu} k^{\mu}\right)^{2} .
\end{gathered}
$$


When considering the vector products in $B(p, k)$, we notice an overall proportionality to $|k|^{2}$. Thus, the condition (66) for the pseudoscalar meson mass has an apparent solution that $m_{p s}^{2}=0$. Once again, a Nambu-Goldstone mode appears as a manifestation of chiral symmetry in this modified NJL model. A second solution for the pseudoscalar meson mass $m_{p s}$ can be found by evaluating the momentum integral in (66) numerically in the Euclidean four-momentum cutoff regularization scheme. Similar to the case of the model based on the vector background field $\Gamma_{a P T_{1}}$ discussed in Sec. IV C, this solution depends on the amplitude of the (pseudovector) background field $B_{\mu}$ and its angle with the pion momentum $k_{\mu}$. Figure 6(a) shows the behavior of this scaled pseudoscalar meson mass as a function of the angle $\alpha$ between the Euclidean background field $B_{E}$ (where $B_{0}=i B_{4}$ ) and the Euclidean meson momentum $k_{E}$ (where $k_{0}=i k_{4}$ ) at fixed values of the coupling constant $\tilde{g}=g\left|B_{E}\right| \Lambda^{-1}$ for which a fermion mass is dynamically generated, i.e., $\tilde{g}<\tilde{g}_{d y n} \approx 1.183$. In Fig. 6(b) the extremal values of the pseudoscalar meson mass in the angular interval are visualized as a function of the coupling constant $\tilde{g}$ in the same region. Since again the mass is a continuous function of $\alpha$, all values between these extrema can be obtained for some value of $\alpha$, which is indicated in Fig. 6(b) as shaded regions. Notice that, independent of the values of $\alpha$ and $\tilde{g}$, the second pseudoscalar mass solution $m_{p s}$ has finite real values.

For the scalar meson mass as determined by condition (65), we do not generally find an apparent solution that factors out of the momentum integral. In particular, we do not find a scalar meson mass that is only proportional to the fermion mass $m$ of the model, in contrast to the solutions of the other non-Hermitian models discussed so far. We also do not generally find a simultaneous solution of the scalar and pseudoscalar meson modes. The two solutions $m_{s}$ that one can find by evaluating (65) numerically in the

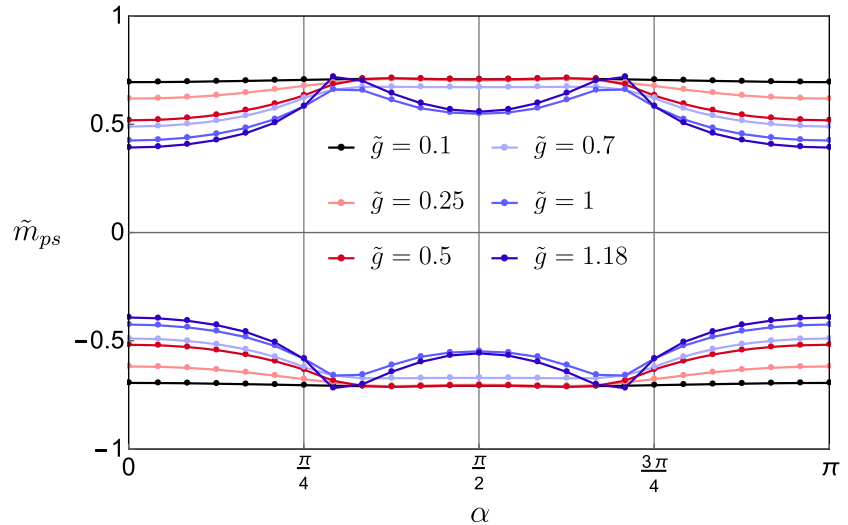

(a)
Euclidean four-momentum cutoff regularization scheme both depend on the angle $\alpha$ between $B_{E}$ and $k_{E}$, and on the value of the scaled coupling constant $\tilde{g}=g\left|B_{E}\right| \Lambda^{-1}$, which is proportional to the amplitude of the background field. Their behavior is shown in Fig. 7(a) as a function of the angle $\alpha$ and for fixed values of $\tilde{g}$. We point out that only for small coupling values below $\tilde{g} \approx 0.107$ do real mass solutions $m_{s}$ exist for all angles $\alpha$. For larger coupling values the solutions around $\alpha=\pi / 2$ begin to break down. However, the region of real masses $m_{s}$ never breaks down entirely and real-valued solutions can always be found for some value of $\alpha$.

The mass of the scalar mode is a continuous function of $\alpha$ and any real mass value between the extremal values over the angle interval is realized at some value of $\alpha$. The behavior of the extremal values is visualized in Fig. 7(b) as a function of coupling constant values for which a fermion mass is dynamically generated $\left(\tilde{g}<\tilde{g}_{d y n} \approx 1.183\right)$. The region of accessible mass values in between minima and maxima is indicated by the shaded area. As remarked above, the two scalar meson mass solutions remain real and separate solutions at all angles $\alpha$ for coupling values below $\tilde{g} \approx 0.107$. For higher coupling values the solutions combine as shown in Fig. 7(a).

Special case $\alpha=0$. We remark that when the pseudovector background field is parallel to the meson momenta, i.e., $\alpha=0$, the contribution $B(p, k)$ in the conditions (65) and (66) vanishes. In this special case we once again find the apparent solutions $m_{p s}^{2}=0$ and $m_{s}^{2}=4 m^{2}$ as in the case of the standard NJL model and the extended model based on the vector field $\Gamma_{a P T_{1}}$.

We further note that $\alpha=0$ is a special case of the underlying modified Dirac theory as well: The dispersion relation of the model with Hamiltonian density

$$
\mathcal{H}=\bar{\psi}\left(-i \gamma^{k} \partial_{k}+i g \gamma_{5} B_{\mu} \gamma^{\mu}\right) \psi
$$

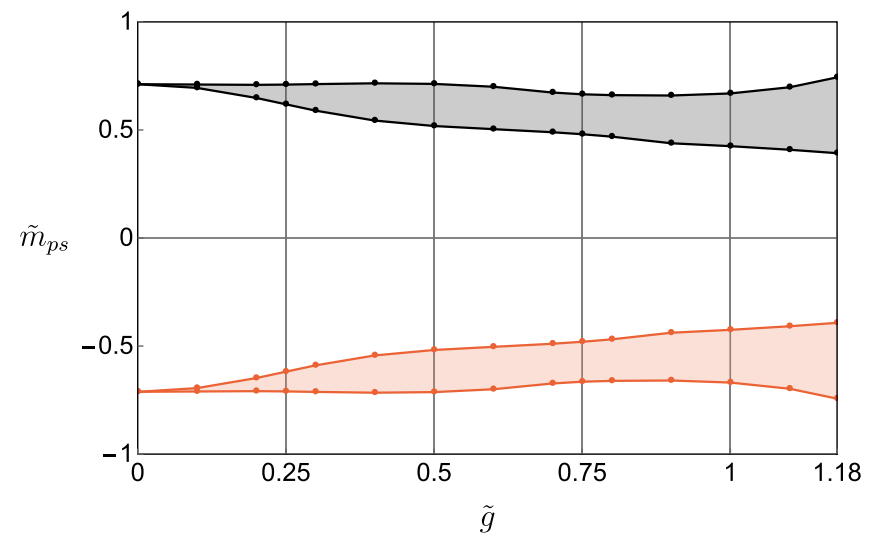

(b)

FIG. 6. In (a) the scaled mass of the pseudoscalar meson $\tilde{m}_{p s}=m_{p s} \Lambda^{-1}$ in the modified NJL model based on $\Gamma_{P T_{1}}$ is shown as function of the angle $\alpha$ between the Euclidean background field and the Euclidean meson momentum for fixed values of the coupling constant $\tilde{g}$. The region of accessible mass over this angle interval is visualized as function of $\tilde{g}$ in (b). 


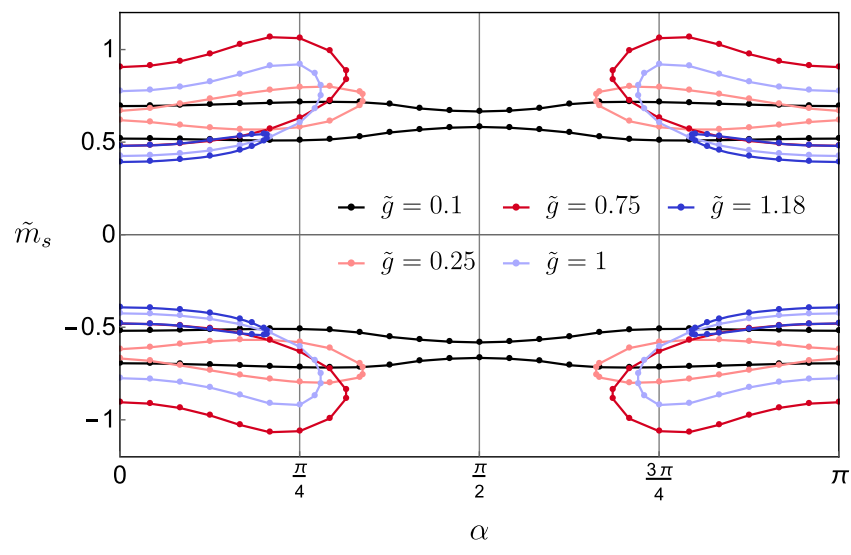

(a)

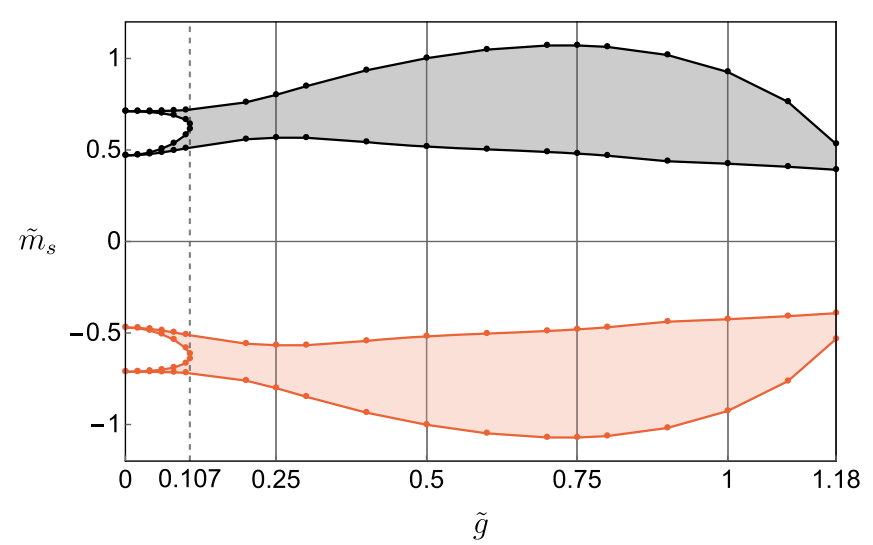

(b)

FIG. 7. In (a) the scaled masses of the scalar meson $\tilde{m}_{s}=m_{s} \Lambda^{-1}$ in the modified NJL model based on $\Gamma_{P T_{1}}$ are shown as function of the angle $\alpha$ between the Euclidean background field and the Euclidean meson momentum for fixed values of the coupling constant $\tilde{g}$. The region of accessible mass over this angle interval is visualized as function of $\tilde{g}$ in (b).

has the form

$$
0=\left(p^{2}-g^{2} B^{2}\right)^{2}+4 g^{2}\left(B_{\mu} p^{\mu}\right)^{2}
$$

at $p^{2}=m^{2}$. Thus, the effective mass of the modified Dirac fermion has the form $m^{2}=g^{2} B^{2} \exp [\mp 2 i \alpha]$. This generally describes complex mass solutions, as noted in [4], but in the special case of $\alpha=0$ we find a purely real fermion mass of $m^{2}=g^{2} B^{2}$.

Overall, we find a Nambu-Goldstone mode for the pseudoscalar meson of this chirally symmetric theory as well as an additional finite pseudoscalar meson mass solution that depends in particular on the angle $\alpha$ between its momentum and the pseudovector background field. Independently, we have found two scalar meson mass solutions which display an intricate dependence on the angle $\alpha$ as well and which, in particular, may break down depending on the specific value of $\alpha$ and the coupling constant $g$.

\section{CONCLUDING REMARKS}

It is surprising that the free Dirac equation in $3+1$ dimensions, augmented by any $\mathcal{P} \mathcal{T}$-symmetric bilinears, does not lead to a real energy spectrum for the fermions unless a bare quark mass is present [4]. (This is counterintuitive, since for bosonic systems, a region in which $\mathcal{P} \mathcal{T}$ symmetry is unbroken leading to a real mass spectrum can usually be found [20].) However, it was shown in [5] that in a fermionic system such as the NJL model, which contains additional two-body interactions, a phase of unbroken $\mathcal{P} \mathcal{T}$ symmetry having real energies can occur within a specific parameter range. In that case, the $\mathcal{P} \mathcal{T}$-symmetric bilinear included in the Hamiltonian density $\mathcal{H}$ given in (2) was $\Gamma_{P T_{1}}$. Having determined that such a Hamiltonian can lead to an additional dynamical mass generation, mimicking the effect of having a bare fermion mass $m_{0}$, we have examined in detail in this paper the effects of including all other possible non-Hermitian bilinear interactions into the Hamiltonian, not only in view of their ability to generate a real fermion spectrum, but also in view of the implications for constructing composite particles. In particular, our interest has been to understand the roles of both $\mathcal{P} \mathcal{T}$ and chiral symmetry, which are both relevant in this context. We have thus considered non-Hermitian scalar $\left(\Gamma_{a P T_{3}}\right)$, pseudoscalar $\left(\Gamma_{a P T_{2}}\right)$, vector $\left(\Gamma_{a P T_{1}}\right)$, pseudovector $\left(\Gamma_{P T_{1}}\right)$, and tensor $\left(\Gamma_{P T_{2}}\right)$ additions to the NJL Hamiltonian.

We find the following results:

(a) $\mathcal{P} \mathcal{T}$ symmetry is not necessary for real fermion mass solutions to exist, rather the two-body interactions supersede the non-Hermitian bilinear effects. To be specific, real solutions for the fermion mass occur for all interactions, with the exception of $\Gamma_{a P T_{3}}$. While the presence of $\Gamma_{P T_{1}}, \Gamma_{a P T_{1}}$, and $\Gamma_{a P T_{2}}$ (pseudovector, vector, and pseudoscalar contributions) can lead to an increase in the dynamically generated mass, mimicking the role of a current quark mass, this is not true when $\Gamma_{P T_{2}}$ is included: then a loss in mass results.

(b) The role of chiral symmetry becomes most evident in the mesonic sector. Both chirally symmetric bilinears, $\Gamma_{a P T_{1}}$ (vector) and $\Gamma_{P T_{1}}$ (pseudovector), as mentioned already in (a), allow for the dynamical generation of fermion masses. In addition, the Goldstone mode remains unaffected, lying again at zero, manifesting the chiral symmetry of the model. For the vector interaction, the scalar (or $\sigma$ ) meson gains mass dynamically as does the fermion, while a second degenerate solution for the composite scalar and pseudoscalar modes depends on the relation of the background field and the meson momentum. It is possible to generate small masses at values of the coupling $\tilde{g}>0.425$, but this again would imply that there is a significant fermion mass. 
For the pseudovector interaction, no general scalar/ pseudoscalar degeneracy is found. Furthermore, the additional pseudoscalar and scalar meson masses depend on the relation of the background field and the meson momentum and remain "large" (of the same size as the degenerate state of the standard NJL model).

The inclusion of a pseudoscalar bilinear leads to a tachionic pseudoscalar mode (which is unphysical) while the scalar mode gains mass dynamically in the same fashion as the fermion, the degenerate solution remaining unaffected.

We thus conclude that the mechanism of mass generation due to four-fermion interactions can occur in many different ways. Non-Hermiticity seems to be no argument for discarding those bilinears that also give rise to the generation of real fermion masses. With the exception of $\Gamma_{a P T_{3}}$, all other forms do this when bare fermion masses are absent. However, in order to give the Goldstone mode a finite mass, another mechanism is required. An additional scalar/pseudoscalar degenerate mode can often be found that is tunable with the coupling strength.

We point out that this analysis is applicable generally to Hamiltonians that include four-fermion interactions, as used for example in discussions of the generation of neutrino masses $[6,7]$.

Finally, we comment on Lorentz symmetry and Lorentzsymmetry breaking as a possible criterion for acceptance of a non-Hermitian model. This is a subtle issue as it depends both on the nature of the fields and on their origin [12-14]. In this work, we have tacitly assumed that both the vector and tensors fields introduced are dynamical. This means that the vector and tensor models studied here are Lorentz invariant, both in the senses of observer and, more subtly, particle invariance. On the other hand, for the case $\Gamma_{P T_{1}}$ the bilinear $i \bar{\psi} \gamma_{5} B_{\mu} \gamma^{\mu} \psi$ forms a Lorentz pseudoscalar, since we have regarded $B_{\mu}$ as a vector. This can be remedied by regarding $B_{\mu}$ as a pseudovector field instead. While it would not change the results of the calculations, which depend only on the magnitude of the field, it would affect its classification as a $\mathcal{P} \mathcal{T}$-symmetry-invariant term. In such a scenario, all terms could be made to be (observer and particle) Lorentz invariant. However, it is thinkable and an open question as to whether a non-Hermitian extension of a high-energy theory would lead, through a symmetrybreaking mechanism to a low-energy Lagrangian thus containing (particle) Lorentz-symmetry breaking terms.
[1] C. M. Bender and S. Boettcher, Phys. Rev. Lett. 80, 5243 (1998).

[2] C. M. Bender, N. Hassanpour, S. P. Klevansky, and S. Sarkar, Phys. Rev. D 98, 125003 (2018).

[3] K. Jones-Smith and H. Mathur, Phys. Rev. A 82, 042101 (2010).

[4] A. Beygi, S. P. Klevansky, and C. M. Bender, Phys. Rev. A 99, 062117 (2019).

[5] A. Felski, A. Beygi, and S. P. Klevansky, Phys. Rev. D 101, 116001 (2020).

[6] J. Alexandre, N. E. Mavromatos, and A. Soto, Nucl. Phys. B961, 115212 (2020).

[7] N. E. Mavromatos and A. Soto, Nucl. Phys. B962, 115275 (2021).

[8] M. N. Chernodub, A. Cortijo, and M. Ruggieri, arXiv:2008 $.11629 \mathrm{v} 1$.

[9] Y. Nambu and G. Jona-Lasinio, Phys. Rev. 122, 345 (1961).

[10] J. Bardeen, L. N. Cooper, and J. R. Schrieffer, Phys. Rev. 108, 1175 (1957).

[11] S. P. Klevansky, Rev. Mod. Phys. 64, 649 (1992).
[12] D. Colloday and V. A. Kostelecký, Phys. Rev. D 55, 6760 (1997).

[13] D. Colloday and V. A. Kostelecký, Phys. Rev. D 58, 116002 (1998).

[14] V. A. Kostelecký and R. Lehnert, Phys. Rev. D 63, 065008 (2001).

[15] J.D. Bjorken and S.D. Drell, Relativistic Quantum Mechanics (McGraw-Hill, New York, 1965).

[16] A. L. Fetter and J.D. Walecka, Quantum Theory of Many-Particle Systems (Dover Publications, New York, 2003).

[17] R. A. Horn and C. R. Johnson, Matrix Analysis, 2nd ed. (Cambridge University Press, Cambridge, England, 2012).

[18] I. S. Gradshteyn and I. M. Ryzhik, Table of Integrals, Series, and Products, 6th ed. (Academic Press, California, 2000).

[19] R. M. Corless, G. H. Gonnet, D. E. G. Hare, D. J. Jeffrey, and D. E. Knuth, Adv. Comput. Math. 5, 329 (1996).

[20] C. M. Bender, PT Symmetry: In Quantum and Classical Physics (World Scientific, Singapore, 2019). 